\title{
Continuation of Normal Doubly Symmetric Orbits in Conservative Reversible Systems.
}

\author{
F.J. Muñoz-Almaraz ${ }^{\star}$, E. Freire ${ }^{\dagger}, J$. Galán ${ }^{\dagger}$ and A. Vanderbauwhede ${ }^{\ddagger}$ \\ *Departamento de Ciencias Físicas, Matemáticas y de la Computación. \\ Universidad Cardenal Herrera-CEU. 46115 Alfara del Patriarca, Spain. \\ ${ }^{\dagger}$ Departamento de Matemática Aplicada II. Escuela Superior de Ingenieros de \\ Sevilla. Camino de los Descubrimientos s/n. Sevilla 41092, Spain. \\ ${ }^{\ddagger}$ Department of Pure Mathematics and Computer Algebra, University of Gent. \\ Krijgslaan 281, B-9000 Gent, Belgium.
}

\begin{abstract}
In this paper we introduce the concept of a quasi-submersive mapping between two finite-dimensional spaces, we obtain the main properties of such mappings, and we introduce "normality conditions" under which a particular class of so-called "constrained mappings" are quasi-submersive at their zeros. Our main application is concerned with the continuation properties of normal doubly symmetric orbits in time-reversible systems with one or more first integrals. As examples we study the continuation of the figure-eight and the supereight choreographies in the N-body problem.
\end{abstract}

Keywords: Hamiltonian and conservative systems; time-reversibility; normal doubly symmetric solutions; periodic solutions; numerical continuation; boundary value problem; $N$-body problem.

\section{Introduction}

It is well known that dynamical systems which are subjected to certain constraints (such as first integrals or symmetries) may show a behavior which is quite different from the generic behavior of a general system without such constraints. A simple example is what happens at an equilibrium where the linearisation of the vector field has a pair of purely imaginary eigenvalues: in the general case one will see a Hopf bifurcation under perturbation of the vector field, while in the case that there is a first integral the system itself (without perturbation) shows a one-parameter family of periodic orbits surrounding the equilibrium (this follows from the Lyapunov Center Theorem and is sometimes referred to as a "vertical Hopf bifurcation"). On a more general level: in general systems periodic orbits are typically isolated, in conservative systems they appear in one- or multi-parameter families.

In this paper we study the existence and continuation of so-called doubly symmetric solutions of reversible systems by studying the solution set of the equations which determine such solutions. It will appear that when the system has one or more first integrals this solution set

(C) 2006 Kluwer Academic Publishers. Printed in the Netherlands. 
forms (locally) a submanifold of an appropriate dimension. To capture the equations which we will encounter in this study in a more general framework we introduce the concept of a "quasi-submersion". As one can expect from its name a quasi-submersion forms a generalization of a submersion in a way which we clarify now using a very simple (and admittedly purely academic) example; still, the example illustrates quite well most of the basic ideas behind the concept.

Consider the mapping $f: \mathbb{R}^{2} \rightarrow \mathbb{R}^{2}$ defined by

$$
f\left(x_{1}, x_{2}\right):=\left(x_{2}-x_{1}^{2}, e^{x_{2}}-e^{x_{1}^{2}}\right), \quad \forall\left(x_{1}, x_{2}\right) \in \mathbb{R}^{2} .
$$

The zeros of $f$ form a one-dimensional curve, namely $x_{2}=x_{1}^{2}$, which is in contrast with the generic situation where one expects isolated zeros. An immediate consequence is that $f$ is not submersive at its zeros. One way to explain this non-generic behaviour is by introducing the mappings $g: \mathbb{R}^{2} \rightarrow \mathbb{R}^{2}, h: \mathbb{R}^{2} \rightarrow \mathbb{R}^{2}$ and $F: \mathbb{R}^{2} \rightarrow \mathbb{R}$ given by

$$
g\left(x_{1}, x_{2}\right):=\left(x_{2}, e^{x_{2}}\right), h\left(x_{1}, x_{2}\right):=\left(x_{1}^{2}, e^{x_{1}^{2}}\right) \text { and } F\left(x_{1}, x_{2}\right):=e^{x_{1}}-x_{2} .
$$

Then $f\left(x_{1}, x_{2}\right)=g\left(x_{1}, x_{2}\right)-h\left(x_{1}, x_{2}\right)$, and the equation $f\left(x_{1}, x_{2}\right)=$ $(0,0)$ is equivalent to the equation $g\left(x_{1}, x_{2}\right)=h\left(x_{1}, x_{2}\right)$. Moreover, we have that

$$
F\left(g\left(x_{1}, x_{2}\right)\right)=F\left(h\left(x_{1}, x_{2}\right)\right), \quad \forall\left(x_{1}, x_{2}\right) \in \mathbb{R}^{2} .
$$

This shows that although the zeros of $f$ are given by the solutions of two equations with two unknowns, there exists a functional relation between these equations, so that in fact we have only one equation in two unknowns leading to a curve of solutions. More formally it follows from (2) that $D F\left(g\left(x_{1}, x_{2}\right)\right) \cdot D g\left(x_{1}, x_{2}\right)=D F\left(h\left(x_{1}, x_{2}\right)\right) \cdot D h\left(x_{1}, x_{2}\right)$, and hence $D F\left(g\left(x_{1}, x_{2}\right)\right) \cdot D f\left(x_{1}, x_{2}\right)=0$ for all zeros $\left(x_{1}, x_{2}\right)$ of $f$. Since $\nabla F\left(x_{1}, x_{2}\right)=\left(e^{x_{1}},-1\right) \neq(0,0)$ this confirms that $f$ can not be a submersion at its zeros. More precisely we have $\operatorname{Im} D f\left(x_{1}, x_{2}\right) \subset$ $\left(\nabla F\left(g\left(x_{1}, x_{2}\right)\right)\right)^{\perp}$ at such zero, and since $D f\left(x_{1}, x_{2}\right) \neq 0$ the inclusion is in fact an equality: $\operatorname{Im} D f\left(x_{1}, x_{2}\right)=\left(\nabla F\left(g\left(x_{1}, x_{2}\right)\right)\right)^{\perp}$ when $f\left(x_{1}, x_{2}\right)=(0,0)$. As will follow from the theory in Section 2 this alone is sufficient to conclude that the zero set of $f$ is (locally) a one-dimensional manifold. One further aspect is that when we take any complementary subspace $W$ of $\operatorname{Im} D f\left(x_{1}, x_{2}\right)$ then there exists a neighborhood $O$ of $\left(x_{1}, x_{2}\right)$ such that $f(O) \cap W=\{(0,0)\}$. This is illustrated in Figure 1 for $\left(x_{1}, x_{2}\right)=(0,0), W=\mathbb{R}(1,0)$ and $O$ the unit disk. Again (see Section 2) this is sufficient to obtain the observed behavior of the zero set of $f$. Finally we observe that these arguments are no longer valid at points $\left(x_{1}, x_{2}\right)$ outside the zero set of $f$; one can directly verify that at such points $f$ is a submersion, except along the 
$x_{2}$-axis where $f$ shows a fold due to its invariance under the symmetry $\left(x_{1}, x_{2}\right) \mapsto\left(-x_{1}, x_{2}\right)$.

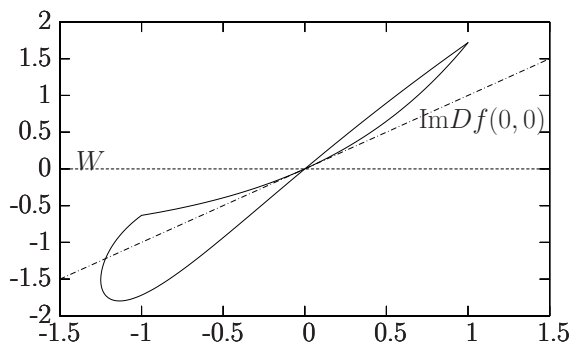

Figure 1. The bounded region delimited by the two closed curves is the range $f(O)$ of the mapping $f$ defined by (1) and with $O$ the unit disk around the origin. The one-dimensional subspace $\operatorname{Im} D f(0,0)$ is generated by the vector $(1,1)$, and the only intersection point of $f(O)$ with the subspace $W$ generated by the vector $(1,0)$ is the point $(0,0)$.

It will appear that the mappings and equations which determine doubly symmetric solutions in reversible systems (see Section 3 for precise definitions) have a similar structure as in the example above, with the "constraints" $F$ corresponding to the first integrals of the system. Therefore we develop in Section 2 a general theory of such mappings, introducing quasi-submersions, constrained mappings and normal zeros. In Section 3 we define doubly symmetric solutions and study their continuation properties. We also set up a boundary value problem which can be used for the numerical continuation of such doubly symmetric solutions. In the final Section 4 we apply our results to the numerical continuation of some special solutions of the $N$-body problem in celestial mechanics; in particular we briefly discuss the figure-eight solution of the 3-body problem (the numerical results for this example can be found in Muñoz Almaraz et al. (2004)), and then turn our attention to the continuation of Gerver's supereight in the 4-body problem. Most of the results in this paper can also be found in the (Spanish) PhD-thesis of the first author (Muñoz Almaraz, 2003).

\section{Quasi-submersions and constrained mappings}

Let $M$ and $N$ be finite dimensional vector spaces, with dimensions respectively $m$ and $n$. Let $f: M \rightarrow N$ be a mapping of class $\mathscr{C}^{r}$ (with $r \geq 1$ ). Given a point $\mathbf{m}_{0} \in M$ we are interested in the local structure (near $\mathbf{m}_{0}$ ) of the set $f^{-1}\left(\left\{f\left(\mathbf{m}_{0}\right)\right\}\right)$. In this section we will describe two sets of conditions which will assure that this set is a submanifold 
of dimension $\operatorname{dim}\left(\operatorname{Ker} D f\left(\mathbf{m}_{0}\right)\right)$. We start by introducing the concept of a quasi-submersion which generalizes that of a submersion. In the subsection on constrained mappings we consider a general situation where the conditions for a quasi-submersion are naturally satisfied. Finally we briefly show how the results from Muñoz Almaraz et al. (2003) fit into the framework described in this section. We should also mention that some of the arguments used here are similar to those used in (Loud and Vanderbauwhede, 1983).

\subsection{QUASI-SUBMERSIONS}

The condition for $f$ to be a submersion at $\mathbf{m}_{0}$ is that $\operatorname{Im}\left(D f\left(\mathbf{m}_{0}\right)\right)=N$; the following definition generalizes this condition.

DEFINITION 1. The function $f$ is a quasi-submersion at $\mathbf{m}_{0}$ iff there exist a neighborhood $O$ of $\mathbf{m}_{0}$ in $M$ and a subspace $W$ of $N$ such that

(QS-1) $W$ is a complement of $\operatorname{Im}\left(D f\left(\mathbf{m}_{0}\right)\right)$ in $N$, i.e.

$$
N=\operatorname{Im}\left(D f\left(\mathbf{m}_{0}\right)\right) \oplus W
$$

(QS-2) the intersection of $f(O)$ and $f\left(\mathbf{m}_{0}\right)+W$ contains only the point $f\left(\mathbf{m}_{0}\right)$, i.e.

$$
f(O) \cap\left(f\left(\mathbf{m}_{0}\right)+W\right)=\left\{f\left(\mathbf{m}_{0}\right)\right\} .
$$

In case $f$ is a submersion at $\mathbf{m}_{0}$ the subspace $W$ is trivial and the condition (QS-2) is void.

A trivial example of a quasi-submersion arises when the mapping $f: M \rightarrow N$ satisfies certain relations, say $\varphi_{i}(f(\mathbf{m}))=0$ for all $\mathbf{m} \in M$, where $\varphi_{i}: N \rightarrow \mathbb{R}(1 \leq i \leq k)$ are $\mathscr{C}^{r}$-mappings such that $\left\{D \varphi_{i}(\mathbf{n}) \mid\right.$ $1 \leq i \leq k\}$ is linearly independent for all $\mathbf{n} \in N$. If $\mathbf{m}_{0} \in M$ is such that $\operatorname{dim} \operatorname{Im}\left(D f\left(\mathbf{m}_{0}\right)\right)=n-k$ (i.e. if $f$ is a submersion at $\mathbf{m}_{0}$ from $M$ onto the codimension $k$ submanifold $N_{0}:=\left\{\mathbf{n} \in N \mid \varphi_{i}(\mathbf{n})=\right.$ $0,1 \leq i \leq k\}$ of $N$ ), then $f: M \rightarrow N$ is trivially a quai-submersion at $\mathbf{m}_{0}$. Another simple example of a quasi-submersion is the following. Suppose that the mapping $f$ can be written as $f(\mathbf{m})=\varphi(\mathbf{m}) g(\mathbf{m})$, with $\varphi: M \rightarrow \mathbb{R}$ and $g: M \rightarrow N$ some $\mathscr{C}^{r}$-mappings. Let $\mathbf{m}_{0} \in M$ be such that $\varphi\left(\mathbf{m}_{0}\right)=0, D \varphi\left(\mathbf{m}_{0}\right) \neq 0$ and $g\left(\mathbf{m}_{0}\right) \neq \mathbf{0}$. Then $\operatorname{Im}\left(D f\left(\mathbf{m}_{0}\right)\right)=$ $\mathbb{R} g\left(\mathbf{m}_{0}\right)$. Fix a complement $W$ of $\mathbb{R} g\left(\mathbf{m}_{0}\right)$ in $N$, and choose a sufficiently small neighborhood $O^{\prime}$ of $g\left(\mathbf{m}_{0}\right)$ in $N$ such that the cone $\mathbb{R} O^{\prime}$ generated by $O^{\prime}$ intersects $W$ only in the origin. Setting $O:=g^{-1}\left(O^{\prime}\right)$ we see that $f(O)$ is contained in the cone $\mathbb{R} O^{\prime}$, and hence $f$ is a quasi-submersion at $\mathbf{m}_{0}$.

The condition (QS-1) implies that

$$
\operatorname{dim}\left(\operatorname{Ker}\left(D f\left(\mathbf{m}_{0}\right)\right)=m-\operatorname{dim}\left(\operatorname{Im}\left(D f\left(\mathbf{m}_{0}\right)\right)=m-n+\operatorname{dim}(W) .\right.\right.
$$


The condition $(\mathbf{Q S - 2})$ means that for all $(\mathbf{m}, \mathbf{w}) \in M \times W$ near $\left(\mathbf{m}_{0}, \mathbf{0}\right)$ we have

$$
f(\mathbf{m})=f\left(\mathbf{m}_{0}\right)+\mathbf{w}
$$

if and only if $\mathbf{m} \in f^{-1}\left(\left\{f\left(\mathbf{m}_{0}\right)\right\}\right)$ and $\mathbf{w}=\mathbf{0}$. From this we easily deduce our main result on quasi-submersions which gives a local description of $f^{-1}\left(\left\{f\left(\mathbf{m}_{0}\right)\right\}\right)$.

THEOREM 2. Suppose the $\mathscr{C}^{r}$-mapping $f: M \rightarrow N$ is a quasisubmersion at $\mathbf{m}_{0} \in M$. Then the set $f^{-1}\left(\left\{f\left(\mathbf{m}_{0}\right)\right\}\right)$ is locally near $\mathbf{m}_{0}$ a $\mathscr{C}^{r}$-submanifold, at $\mathbf{m}_{0}$ tangent to $\operatorname{Ker}\left(D f\left(\mathbf{m}_{0}\right)\right)$ and with dimension equal to $\operatorname{dim}\left(\operatorname{Ker}\left(D f\left(\mathbf{m}_{0}\right)\right)\right)$.

Proof. Using the implicit function theorem his follows immediately from the foregoing observations and from the fact that the mapping

$$
\varphi: M \times W \longrightarrow N,(\mathbf{m}, \mathbf{w}) \longmapsto \varphi(\mathbf{m}, \mathbf{w}):=f(\mathbf{m})-\mathbf{w}
$$

is a submersion at $\left(\mathbf{m}_{0}, \mathbf{0}\right)$.

Remark 1. Since both the foregoing definition and theorem are of a local nature it is straightforward to generalize them to the situation where $M$ and $N$ are (finite-dimensional) manifolds, and $W$ a submanifold of $N$. For a generalization to mappings between Banach spaces we refer to (Muñoz Almaraz, 2003).

The main difficulty in using the concept of a quasi-submersion consists of course in verifying the condition (3) for an appropriate neighborhood $O$ of the point $\mathbf{m}_{0} \in M$ under consideration. Motivated by our main application (which has to do with certain solutions of autonomous systems having one or more first integrals) we describe in the next subsection a situation where the condition (3) is "typically" satisfied at most zeros of the mapping $f$.

\subsection{Constrained mappings}

In this subsection we restrict ourselves to mappings $f: M \rightarrow N$ which can be written in the form $f=g-h$, where $g: M \rightarrow N$ and $h: M \rightarrow N$ are $\mathscr{C}^{r}$-mappings, with $r \geq 1$. We also assume that there exist one or more (non-constant) $\mathscr{C}^{1}$-functions $F: N \rightarrow \mathbb{R}$ such that

$$
F(g(\mathbf{m}))=F(h(\mathbf{m})), \quad \forall \mathbf{m} \in M .
$$

Clearly the set of all such constraining functions (including the constant ones) forms a vectorspace which we denote by $\mathscr{F}$. For convenience of formulation we will call a mapping $f$ satisfying the foregoing conditions a constrained mapping. Finally we will restrict ourselves to the 
case where $\mathbf{m}_{0}$ is a zero of $f$, i.e. $g\left(\mathbf{m}_{0}\right)=h\left(\mathbf{m}_{0}\right)$; we set $\mathbf{n}_{0}:=$ $g\left(\mathbf{m}_{0}\right)=h\left(\mathbf{m}_{0}\right)$. Studying $f^{-1}\left(\left\{f\left(\mathbf{m}_{0}\right)\right\}\right)$ near $\mathbf{m}_{0}$ means then that we are interested in the continuation of the solution $\mathbf{m}_{0}$ of the equation

$$
g(\mathbf{m})=h(\mathbf{m}) .
$$

Remark 2. Again all our results will be local, and therefore the assumption that (6) should be satisfied for all $\mathbf{m} \in M$ is unnecessarily strong; it is sufficient for (6) to hold for all $\mathbf{m}$ in a neighborhood of $\mathbf{m}_{0}$. A more formal definition of $\mathscr{F}$ would then take the following form: $\mathscr{F}$ is the space of 1 -jets at $\mathbf{n}_{0}$ of all $\mathscr{C}^{1}$-functions $F: N \rightarrow \mathbb{R}$ such that $F(g(\mathbf{m}))=F(h(\mathbf{m}))$ for all $\mathbf{m}$ in some neighborhood of $\mathbf{m}_{0}$.

Differentiating (6) at $\mathbf{m}=\mathbf{m}_{0}$ shows that

$$
D F\left(\mathbf{n}_{0}\right) \cdot D g\left(\mathbf{m}_{0}\right)=D F\left(\mathbf{n}_{0}\right) \cdot D h\left(\mathbf{m}_{0}\right),
$$

and hence $D F\left(\mathbf{n}_{0}\right) \cdot D f\left(\mathbf{m}_{0}\right)=0$, for all $F \in \mathscr{F}$. This proves the following.

LEMMA 3. Let $\mathbf{m}_{0}$ be a zero of a constrained $\mathscr{C}^{r}$-mapping $f$, as described above. Then

$$
\operatorname{Im} D f\left(\mathbf{m}_{0}\right) \subset \mathscr{W}^{\perp}:=\bigcap_{F \in \mathscr{F}} \operatorname{Ker}\left(D F\left(\mathbf{n}_{0}\right)\right) .
$$

Observe that $\mathscr{W}^{\perp}$ is the annihilator of $\mathscr{W}:=\left\{D F\left(\mathbf{n}_{0}\right) \mid F \in \mathscr{F}\right\} \subset$ $\mathcal{L}(N ; \mathbb{R})$. The inclusion (8) suggests the following definition.

DEFINITION 4. The zero $\mathbf{m}_{0}$ of the constrained mapping $f$ is called normal if we have equality in (8), i.e. if

$$
\operatorname{Im} D f\left(\mathbf{m}_{0}\right)=\mathscr{W}^{\perp}=\bigcap_{F \in \mathscr{F}} \operatorname{Ker}\left(D F\left(\mathbf{n}_{0}\right)\right) .
$$

As we will show in subsection 2.3 the normality concept introduced here encompasses the concept of a normal periodic orbit in systems with first integrals as defined in (Muñoz Almaraz et al., 2003), which itself was a generalization of an idea first introduced by Sepulchre and MacKay (1997). One expects that for a "typical" constrained mapping $f$ the normality condition (9) will be satisfied at most of its zeros. Without proving anything in this direction we can at least affirm that in the examples which we have studied this seems to be indeed the case.

Our main result on constrained mappings is the following. 
THEOREM 5. A constrained mapping $f: M \rightarrow N$ is a quasi-submersion at each of its normal zeros. In particular, if $\mathbf{m}_{0} \in M$ is a normal zero of $f=g-h$, then the set

$$
\{\mathbf{m} \in M \mid g(\mathbf{m})=h(\mathbf{m})\}
$$

is close to $\mathbf{m}_{0}$ a submanifold, with dimension equal to

$\operatorname{dim} \operatorname{Ker}\left(D f\left(\mathbf{m}_{0}\right)\right)=m-\operatorname{dim} \operatorname{Im}\left(D f\left(\mathbf{m}_{0}\right)\right)=m-n+\operatorname{codim}\left(\mathscr{W}^{\perp}\right)$.

Proof. Let $W$ be a complement of $\mathscr{W}^{\perp}$ in $N$, such that $N=\mathscr{W}^{\perp} \oplus$ $W=\operatorname{Im}\left(D f\left(\mathbf{m}_{0}\right)\right) \oplus W$. Since $f\left(\mathbf{m}_{0}\right)=\mathbf{0}$ we have to show that there exists a neighborhood $O$ of $\mathbf{m}_{0}$ in $M$ such that $f(O) \cap W=\{\mathbf{0}\}$. To do so we choose $F_{i} \in \mathscr{F}\left(1 \leq i \leq k:=\operatorname{codim}\left(\mathscr{W}^{\perp}\right)=\operatorname{dim} W\right)$ such that $\left\{D F_{i}\left(\mathbf{n}_{0}\right) \mid 1 \leq i \leq k\right\}$ forms a basis of $\mathscr{W}=\left\{D F\left(\mathbf{n}_{0}\right) \mid F \in \mathscr{F}\right\}$. Also, let $P \in \mathcal{L}(N)$ be the linear projection in $N$ such that $\operatorname{Im} P=\mathscr{W}^{\perp}$ and Ker $P=W$ (remember that $N=\mathscr{W}^{\perp} \oplus W$ ).

Now suppose that $\mathbf{m} \in M$ is such that $f(\mathbf{m})=g(\mathbf{m})-h(\mathbf{m}) \in W$. Then $\operatorname{Pf}(\mathbf{m})=\mathbf{0}$, or, written differently, $P g(\mathbf{m})=P h(\mathbf{m})$. Combining this with (6) (valid for all $F \in \mathscr{F}$, and in particular for $F=F_{i}$, $1 \leq i \leq k)$ we conclude that

$$
\Phi(g(\mathbf{m}))=\Phi(h(\mathbf{m})),
$$

where $\Phi: N \rightarrow \mathscr{W}^{\perp} \oplus \mathbb{R}^{k}$ is defined by

$$
\Phi(\mathbf{n}):=\left(P \mathbf{n}, F_{1}(\mathbf{n}), \ldots, F_{k}(\mathbf{n})\right), \quad \forall \mathbf{n} \in N .
$$

We claim that $\Phi$ is a local $\mathscr{C}^{1}$-diffeomorphism near $\mathbf{n}_{0}$. Indeed, it follows from the definitions that $N$ and $\mathscr{W}^{\perp} \oplus \mathbb{R}^{k}$ have the same dimension, while for each $\mathbf{n} \in N$ the equality

$$
D \Phi\left(\mathbf{n}_{0}\right) \cdot \mathbf{n}=\left(P \mathbf{n}, D F_{1}\left(\mathbf{n}_{0}\right) \cdot \mathbf{n}, \ldots, D F_{k}\left(\mathbf{n}_{0}\right) \cdot \mathbf{n}\right)=(\mathbf{0}, 0, \ldots, 0)
$$

implies that

$$
\mathbf{n} \in \operatorname{Ker} P \cap\left(\cap_{1 \leq i \leq k} \operatorname{Ker} D F_{i}\left(\mathbf{n}_{0}\right)\right)=W \cap \mathscr{W}^{\perp}=\{\mathbf{0}\} .
$$

We conclude that if $\mathbf{m} \in M$ is sufficiently close to $\mathbf{m}_{0}$ and satisfies (10), then $g(\mathbf{m})=h(\mathbf{m})$ and $f(\mathbf{m})=\mathbf{0}$. It follows that $f$ is a quasisubmersion at $\mathbf{m}_{0}$, and the remaining part of the theorem follows directly from Theorem 2 .

It is possible to make the choice of the complement $W$ of $\mathscr{W}^{\perp}$ in the foregoing proof a bit more explicit by introducing an inner product $\langle\cdot, \cdot\rangle$ in $N$. Then we denote by $\nabla F: N \rightarrow N$ the corresponding gradient 
of a $\mathscr{C}^{1}$-function $F: N \rightarrow \mathbb{R}$, such that $D F(\mathbf{n}) \cdot \tilde{\mathbf{n}}=\langle\nabla F(\mathbf{n}), \tilde{\mathbf{n}}\rangle$ for all $\mathbf{n}, \tilde{\mathbf{n}} \in N$. With $\mathscr{F}$ defined as before we set

$$
W:=\left\{\nabla F\left(\mathbf{n}_{0}\right) \mid F \in \mathscr{F}\right\},
$$

and we choose $F_{i} \in \mathscr{F}(1 \leq i \leq k)$ such that $\left\{\nabla F_{i}\left(\mathbf{n}_{0}\right) \mid 1 \leq i \leq k\right\}$ forms a basis of $W$. It is then easy to see that

$$
\mathscr{W}^{\perp}=\{\mathbf{n} \in N \mid\langle\mathbf{w}, \mathbf{n}\rangle=\mathbf{0}, \forall \mathbf{w} \in W\}=W^{\perp} .
$$

It follows that $W$ is indeed a complement of $\mathscr{W}^{\perp}$ in $N$, and that $\left\{D F_{i}\left(\mathbf{n}_{0}\right) \mid 1 \leq i \leq k\right\}$ forms a basis of $\mathscr{W}$. Combining this with the proof of Theorem 2 gives us a method to compute the zero set of $f$ near $\mathbf{m}_{0}$, as follows.

COROLLARY 6. Using the foregoing notations, let $\mathbf{m}_{0} \in M$ be a normal zero of the constrained mapping $f=g-h$. Then the mapping $\phi: M \times \mathbb{R}^{k} \rightarrow N$ defined by

$$
\phi(\mathbf{m}, \alpha):=f(\mathbf{m})+\sum_{1 \leq i \leq k} \alpha_{i} \nabla F_{i}\left(\mathbf{n}_{0}\right), \quad\left(\alpha=\left(\alpha_{1}, \ldots, \alpha_{k}\right) \in \mathbb{R}^{k}\right),
$$

is a submersion at $(\mathbf{m}, \alpha)=\left(\mathbf{m}_{0}, \mathbf{0}\right)$, and one can apply the implicit function theorem to solve the equation

$$
f(\mathbf{m})+\sum_{1 \leq i \leq k} \alpha_{i} \nabla F_{i}\left(\mathbf{n}_{0}\right)=\mathbf{0} .
$$

For all solutions of this equation near $(\mathbf{m}, \alpha)=\left(\mathbf{m}_{0}, \mathbf{0}\right)$ we have that $\alpha=\mathbf{0}$, and hence these solutions determine the zero set of $f$ near $\mathbf{m}_{0}$.

From (8) and the fact that the mapping $\mathbf{m} \mapsto \operatorname{dim} \operatorname{Im} D f(\mathbf{m})$ is lower-semicontinuous we obtain the following corollary of Theorem 5 .

COROLLARY 7. Under the conditions of Theorem 5 all zeros of $f$ sufficiently close to $\mathbf{m}_{0}$ are also normal.

\subsection{An EXAMPLE: PERIODIC ORBITS IN CONSERVATIVE SySTEMS}

In this subsection we briefly show how the concept of a normal periodic orbit in a system with first integrals as defined in Muñoz Almaraz et al. (2003) fits into the framework described in the preceding subsection.

Consider the system $\dot{\mathbf{x}}=X(\mathbf{x})$, with $X: \mathbb{R}^{n} \rightarrow \mathbb{R}^{n}$ a vectorfield of class $\mathscr{C}^{r}(r \geq 1)$. Denote by $\mathscr{F}$ the space of first integrals of $X$, i.e. the space of $\mathscr{C}^{1}$-functions $F: \mathbb{R}^{n} \rightarrow \mathbb{R}$ such that $D F(\mathbf{x}) \cdot X(\mathbf{x})=0$ for 
all $\mathbf{x} \in \mathbb{R}^{n}$. Also, let $\tilde{\mathbf{x}}(t, \mathbf{x})$ denote the flow of $X$. Nontrivial periodic orbits of $X$ are given by the solutions $\left(T, \mathbf{x}_{0}\right) \in \mathbb{R} \times \mathbb{R}^{n}$ of the equation

$$
\tilde{\mathbf{x}}(t, \mathbf{x})=\mathbf{x}
$$

with $T>0$ and $X\left(\mathbf{x}_{0}\right) \neq \mathbf{0}$. Then $\tilde{\mathbf{x}}\left(t, \mathbf{x}_{\mathbf{0}}\right)$ is a $T$-periodic solution of $\dot{\mathbf{x}}=X(\mathbf{x})$; for simplicity we will assume that $T$ is minimal. The equation (12) is of the form (7) when we set $M:=\mathbb{R} \times \mathbb{R}^{n}, N:=\mathbb{R}^{n}$, and define $g, h: \mathbb{R} \times \mathbb{R}^{n} \rightarrow \mathbb{R}^{n}$ by

$$
g(t, \mathbf{x}):=\tilde{\mathbf{x}}(t, \mathbf{x}) \quad \text { and } \quad h(t, \mathbf{x}):=\mathbf{x} .
$$

Clearly (6) is satisfied for all first integrals $F \in \mathscr{F}$. If $\mathbf{m}_{0}=\left(T, \mathbf{x}_{0}\right)$ is a solution of (12) then $\mathbf{n}_{0}=\mathbf{x}_{0}$ and (with $f:=g-h$ )

$$
D f\left(T, \mathbf{x}_{0}\right) \cdot(t, \mathbf{x})=(M-I) \cdot \mathbf{x}+t X\left(\mathbf{x}_{0}\right), \quad \forall(t, \mathbf{x}) \in \mathbb{R} \times \mathbb{R}^{n} ;
$$

here $M \in \mathcal{L}\left(\mathbb{R}^{n}\right)$ is the monodromy matrix of the periodic orbit generated by the point $\mathbf{x}_{0}$, i.e. $M=V(T)$, with $V: \mathbb{R} \rightarrow \mathcal{L}\left(\mathbb{R}^{n}\right)$ the solution of the initial value problem

$$
\dot{V}(t)=D X\left(\tilde{\mathbf{x}}\left(t, \mathbf{x}_{0}\right)\right) \cdot V(t), \quad V(0)=I .
$$

Using the standard scalar product on $\mathbb{R}^{n}$ and setting $W:=\left\{\nabla F\left(\mathbf{x}_{0}\right) \mid\right.$ $F \in \mathscr{F}\}$ it follows from the foregoing general theory that

$$
\operatorname{Im} D f\left(T, \mathbf{x}_{0}\right)=\operatorname{Im}(M-I)+\mathbb{R} X\left(\mathbf{x}_{0}\right) \subset W^{\perp} .
$$

By the definition given in (Muñoz Almaraz et al., 2003) the periodic orbit generated by $\mathbf{x}_{0}$ is called normal if we have equality in the foregoing inclusion, i.e. if

$$
\operatorname{Im}(M-I)+\mathbb{R} X\left(\mathbf{x}_{0}\right)=W^{\perp} .
$$

We conclude that the periodic orbit generated by the solution $\left(T, \mathbf{x}_{0}\right)$ of $(12)$ is normal if and only if $\left(T, \mathbf{x}_{0}\right)$ is a normal zero of the constrained mapping $f$ associated with the equation (12). By the foregoing theory, using the fact that all points on a periodic orbit form a onedimensional curve of solutions of $(12)$, and setting $k:=\operatorname{dim}(W)$ we conclude that a normal periodic orbit of the conservative vectorfield $X$ belongs to a $[(n+1)-(n-k)-1]=k$-parameter family of (normal) periodic orbits. This is precisely the continuation result obtained in (Muñoz Almaraz et al., 2003). 


\section{Doubly symmetric solutions in reversible systems}

In this section we consider autonomous and finite-dimensional systems of the form

$$
\dot{\mathbf{x}}=X(\mathbf{x}),
$$

with $\mathbf{x} \in \mathbb{R}^{n}$ and $X: \mathbb{R}^{n} \rightarrow \mathbb{R}^{n}$ a smooth vector field. We denote the flow of $(13)$ by $\tilde{\mathbf{x}}(t, \mathbf{x})$; this flow is defined and smooth for $(t, \mathbf{x})$ in an appropriate open subset of $\mathbb{R} \times \mathbb{R}^{n}$. The system (13) is time-reversible (or reversible for short) if there exist a subgroup $\Gamma$ of the orthogonal group $O(n)$ and a nontrivial character $\chi: \Gamma \rightarrow\{1,-1\}$ such that

$$
X(\gamma \cdot \mathbf{x})=\chi(\gamma) \gamma \cdot X(\mathbf{x}), \quad \forall \gamma \in \Gamma .
$$

The flow will then be such that

$$
\tilde{\mathbf{x}}(\chi(\gamma) t, \gamma \cdot \mathbf{x})=\gamma \cdot \tilde{\mathbf{x}}(t, \mathbf{x}), \quad \forall \gamma \in \Gamma, \forall(t, \mathbf{x})
$$

The system (13) may also have one or more first integrals. Such type of system appears frequently in mechanics, and in particular in celestial mechanics (see (Howison and Meyer, 2000) and Section 4 of this paper for some examples). For a survey on reversible systems with many further references, see (Lamb and Roberts, 1998).

In the foregoing set-up with symmetries it is natural to look for solutions and orbits which show some kind of symmetry. In the next subsection we will study a particular kind of such solutions, called "doubly symmetric solutions". The main results of this section are found in subsection 3.2 where we use the results of Section 2 to study the continuation of doubly symmetric solutions; in this subsection we introduce the concept of a normal doubly symmetric solution, analogous to that of a normal periodic orbit as found in (Muñoz Almaraz et al., 2003) and strongly related to that of a normal zero of constrained mappings. In subsection 3.3 we describe a boundary value problem which can be used for the numerical continuation of doubly symmetric solutions. In subsection 3.4 we consider the continuation of doubly symmetric solutions in the case of Hamiltonian systems, thereby providing an introduction to the applications in Section 4. The importance of these doubly symmetric solutions in the $N$-body problem, and in particular in the study of choreographies, was already emphasized in (Marchal, 2000), and will be further illustrated in Section 4. Also, see (Meyer, 1998) and (Howison and Meyer, 2000) for examples in the restricted and reduced 3-body problem. 


\subsection{Doubly Symmetric SOlutions}

We are interested in solutions of (13) which show some symmetry with respect to two (given or chosen) reversors of (13), which we will denote by $R_{0}$ and $R_{1}$; a reversor is an element of $\Gamma$ for which the corresponding character equals -1 . So we assume that $R_{0}, R_{1} \in O(n)$ and that

$$
X\left(R_{0} \mathbf{x}\right)=-R_{0} X(\mathbf{x}) \quad \text { and } \quad X\left(R_{1} \mathbf{x}\right)=-R_{1} X(\mathbf{x}) .
$$

Observe from the onset that the case $R_{1}=R_{0}$ is allowed. For each $\gamma \in \Gamma$ we denote by $\operatorname{Fix}(\gamma):=\left\{\mathbf{x} \in \mathbb{R}^{n} \mid \gamma \cdot \mathbf{x}=\mathbf{x}\right\}$ the fixed point subspace of $\gamma$. We start with a few properties of the solutions of (13) which follow from (16) and (15):

Property 1. If $\hat{\mathrm{x}}: J \subset \mathbb{R} \rightarrow \mathbb{R}^{n}$ is a maximal solution of (13) such that $\hat{\mathbf{x}}\left(t_{0}\right) \in \operatorname{Fix}(R)$ for some $t_{0} \in J$ and with $R=R_{0}$ or $R_{1}$, then we have for all $\tau \in \mathbb{R}$ that $\left(t_{0}+\tau\right) \in J$ if and only if $\left(t_{0}-\tau\right) \in J$, and for such $\tau$ the following holds:

$$
R \hat{\mathbf{x}}\left(t_{0}-\tau\right)=\hat{\mathbf{x}}\left(t_{0}+\tau\right) .
$$

Property 2. If $\hat{\mathbf{x}}: J \subset \mathbb{R} \rightarrow \mathbb{R}^{n}$ is a maximal solution of (13) such that $\hat{\mathbf{x}}\left(t_{1}\right)=S \hat{\mathbf{x}}\left(t_{0}\right)$ for some $t_{0}, t_{1} \in J\left(t_{1} \neq t_{0}\right)$ and with $S=R_{0} R_{1}$ or $R_{1} R_{0}$, then $J=\mathbb{R}$ and we have for all $\tau \in \mathbb{R}$ that

$$
\hat{\mathbf{x}}\left(t_{1}+\tau\right)=S \hat{\mathbf{x}}\left(t_{0}+\tau\right) .
$$

(This follows from $X(S \mathbf{x})=S X(\mathbf{x})$ ).

Next we come to the definition of doubly symmetric solutions.

DEFINITION 8. A solution $\hat{\mathbf{x}}: J \subset \mathbb{R} \rightarrow \mathbb{R}^{n}$ of $(13)$ is called $\left(R_{0}, R_{1}\right)$ symmetric, or doubly symmetric with respect to $\left(R_{0}, R_{1}\right)$ if there exist $t_{0}, t_{1} \in J$ with $T:=t_{1}-t_{0}>0$ and such that

$$
\hat{\mathbf{x}}\left(t_{0}\right) \in \operatorname{Fix}\left(R_{0}\right) \quad \text { and } \quad \hat{\mathbf{x}}\left(t_{1}\right) \in \operatorname{Fix}\left(R_{1}\right) .
$$

We call $\left[t_{0}, t_{1}\right]$ the basic domain of the $\left(R_{0}, R_{1}\right)$-symmetric solution $\hat{\mathbf{x}}(t)$.

By a phase shift we can without loss of generality assume that $t_{0}=0$ and $t_{1}=T$, i.e. that $[0, T]$ forms the basic domain. In many examples (see e.g. Section 4$)$ it will appear that $\left(R_{0}, R_{1}\right)$-symmetric solutions are also periodic, with a period which is a multiple of $T$; in such cases $T$ as used here should not be confused with the (minimal) period. Also, it follows from Property 1 above that $\hat{\mathbf{x}}(t) \in \operatorname{Fix}\left(R_{0}^{2}\right) \cap \operatorname{Fix}\left(R_{1}^{2}\right)$ for all $t \in J$; hence, by restricting the system (13) to the invariant subspace $\operatorname{Fix}\left(R_{0}^{2}\right) \cap \operatorname{Fix}\left(R_{1}^{2}\right)$, we can (and will) without loss of generality assume that $R_{0}^{2}=R_{1}^{2}=I$.

One of the basic properties of doubly symmetric solutions is the following. 
LEMMA 9. Let $\hat{\mathbf{x}}: J \subset \mathbb{R} \rightarrow \mathbb{R}^{n}$ be an $\left(R_{0}, R_{1}\right)$-symmetric solution. Then this solution can be extended to the whole real line, and, assuming the basic domain is $[0, T]$, we have for all $t \in \mathbb{R}$ and all $m \in \mathbb{Z}$ that

$$
\begin{aligned}
& \hat{\mathbf{x}}(-t)=R_{0} \hat{\mathbf{x}}(t), \quad \hat{\mathbf{x}}(T-t)=R_{1} \hat{\mathbf{x}}(T+t) \quad \text { and } \\
& \hat{\mathbf{x}}(2 m T+t)=\left(R_{1} R_{0}\right)^{m} \hat{\mathbf{x}}(t) .
\end{aligned}
$$

Observe that the relations (18) allow to reconstruct the full solution $\hat{\mathbf{x}}: \mathbb{R} \rightarrow \mathbb{R}^{n}$ by applying the appropriate symmetry operations to the restriction of $\hat{\mathbf{x}}$ to its basic domain $[0, T]$. Also, the relations (18) can be easily adapted to the case of a general basic domain $\left[t_{0}, t_{1}\right]$.

Proof. Let $\hat{\mathbf{x}}: J \rightarrow \mathbb{R}^{n}$ be a maximal $\left(R_{0}, R_{1}\right)$-symmetric solution, with basic domain $\left[t_{0}, t_{1}\right]$. Then, by Property 1 above, $J$ must be an open interval symmetric about both $t_{0}$ and $t_{1}$; this is only possible with $J=\mathbb{R}$. Assuming the basic domain is $[0, T]$ the first two relations of (18) follow from Property 1 (take $\left(R, t_{0}\right)=\left(R_{0}, 0\right)$ and $\left(R, t_{0}\right)=$ $\left(R_{1}, T\right)$, respectively). Setting $t=-T$ in the second relation we find that $\hat{\mathbf{x}}(2 T)=R_{1} \hat{\mathbf{x}}(0)=R_{1} R_{0} \hat{\mathbf{x}}(0)$, and then the last relation in (18) follows by applying Property 2 above repeatedly (with $S=R_{1} R_{0}$ and $\left.\left(t_{1}, t_{0}, \tau\right)=(2 m T, 2(m-1) T, t)\right)$.

It follows from the last relation in (18) that if $\left(R_{1} R_{0}\right)^{M}=I$ for some $M>0$ then an $\left(R_{0}, R_{1}\right)$-symmetric solution will be automatically periodic, with period $2 M T$ (see (Howison and Meyer, 2000) for an example). In particular this will be the case when $R_{1}=R_{0}$; since then $R_{1} R_{0}=R_{0}^{2}=I$ we get the following corollary of Lemma 9 .

COROLLARY 10. Each $\left(R_{0}, R_{0}\right)$-symmetric solution of $(13)$ is periodic, with period equal to twice the length of the basic domain. Moreover, the orbit of such solution is invariant under $R_{0}$.

Such $\left(R_{0}, R_{0}\right)$-symmetric solutions are called symmetric periodic solutions (or $R_{0}$-symmetric periodic orbits if we want to indicate the symmetry $R_{0}$ ). They are the only doubly symmetric solutions which can appear when the group $\Gamma$ is minimal, i.e. when $\Gamma=\left\{I, R_{0}\right\}$, with $R_{0}^{2}=I$ and $X\left(R_{0} \mathbf{x}\right)=-R_{0} X(\mathbf{x})$.

COROLLARY 11. Let $\hat{\mathbf{x}}: \mathbb{R} \rightarrow \mathbb{R}^{n}$ be a $\left(R_{0}, R_{1}\right)$-symmetric solution with basic domain $[0, T]$. Then $\hat{\mathbf{x}}$ is for each $\ell \in \mathbb{Z}$ also a doubly symmetric solution with respect to $\left(\left(R_{1} R_{0}\right)^{\ell-1} R_{1},\left(R_{1} R_{0}\right)^{\ell} R_{1}\right)$, with basic domain $[\ell T,(\ell+1) T]$.

Proof. It follows from (18) that for each $m \in \mathbb{Z}$ we have that

$$
\begin{aligned}
\hat{\mathbf{x}}(2 m T) & =\left(R_{1} R_{0}\right)^{m} \hat{\mathbf{x}}(0)=\left(R_{1} R_{0}\right)^{m} R_{0} \hat{\mathbf{x}}(0) \\
& =\left(R_{1} R_{0}\right)^{m} R_{0}\left(R_{0} R_{1}\right)^{m} \hat{\mathbf{x}}(2 m T)=\left(R_{1} R_{0}\right)^{2 m-1} R_{1} \hat{\mathbf{x}}(2 m T)
\end{aligned}
$$


and

$$
\begin{aligned}
& \hat{\mathbf{x}}((2 m+1) T)=\left(R_{1} R_{0}\right)^{m} \hat{\mathbf{x}}(T)=\left(R_{1} R_{0}\right)^{m} R_{1} \hat{\mathbf{x}}(T) \\
& \quad=\left(R_{1} R_{0}\right)^{m} R_{1}\left(R_{0} R_{1}\right)^{m} \hat{\mathbf{x}}((2 m+1) T)=\left(R_{1} R_{0}\right)^{2 m} R_{1} \hat{\mathbf{x}}((2 m+1) T) .
\end{aligned}
$$

Setting $\ell=2 m$ or $\ell=2 m+1$ this shows that

$$
\hat{\mathbf{x}}(\ell T)=\left(R_{1} R_{0}\right)^{\ell-1} R_{1} \hat{\mathbf{x}}(\ell T),
$$

which proves the corollary.

In case $\left(R_{1} R_{0}\right)^{M}=I$ we can not only consider $\hat{\mathbf{x}}(t)$ as a $2 M T$ periodic solution, but also as a $\left(R_{0}, R_{0}\right)$-symmetric solution with basic domain $[0, M T]$, or as a $\left(R_{1}, R_{1}\right)$-symmetric solution with basic domain $[T,(M+1) T]$; this follows from (19) by taking $\ell=M$ or $\ell=M+1$.

\subsection{Continuation of Doubly Symmetric SOlutions}

We are mainly interested in the continuation of doubly symmetric solutions, i.e. we want to answer the following type of question: given a doubly symmetric solution, does it belong to a one- or more-parameter family of doubly symmetric solutions, and how can we calculate this family? In order to study this problem we need to set up equations from which doubly symmetric solutions can be determined. First we introduce some notation. It follows from $R_{0}^{2}=R_{1}^{2}=I$ that

$$
\mathbb{R}^{n}=\operatorname{Fix}\left(R_{0}\right) \oplus \operatorname{Fix}\left(-R_{0}\right)=\operatorname{Fix}\left(R_{1}\right) \oplus \operatorname{Fix}\left(-R_{1}\right) .
$$

We define projections onto these fixed point subspaces as follows:

$$
\pi_{0}^{ \pm}:=\frac{1}{2}\left(I \pm R_{0}\right), \quad \pi_{1}^{ \pm}:=\frac{1}{2}\left(I \pm R_{1}\right) .
$$

Then $\operatorname{Im} \pi_{0}^{ \pm}=\operatorname{Ker} \pi_{0}^{\mp}=\operatorname{Fix}\left( \pm R_{0}\right)$ and $\operatorname{Im} \pi_{1}^{ \pm}=\operatorname{Ker} \pi_{1}^{\mp}=\operatorname{Fix}\left( \pm R_{1}\right)$. We also set $n_{0}^{ \pm}:=\operatorname{dim}\left(\operatorname{Fix}\left( \pm R_{0}\right)\right)$ and $n_{1}^{ \pm}:=\operatorname{dim}\left(\operatorname{Fix}\left( \pm R_{1}\right)\right)$. In many examples we have that $n_{0}^{+}=n_{0}^{-}=n_{1}^{+}=n_{1}^{-}$, but we will not make that assumption here.

An initial point $\mathbf{x}_{0} \in \mathbb{R}^{n}$ will generate an $\left(R_{0}, R_{1}\right)$-symmetric solution $\tilde{\mathbf{x}}\left(t, \mathbf{x}_{0}\right)$ with basic domain $[0, T]$ if and only if $\mathbf{x}_{0} \in \operatorname{Fix}\left(R_{0}\right)$ and $\tilde{\mathbf{x}}\left(T, \mathbf{x}_{0}\right) \in \operatorname{Fix}\left(R_{1}\right)$. Such points correspond to the zeros of the mapping $f: \mathbb{R} \times \operatorname{Fix}\left(R_{0}\right) \rightarrow \mathbb{R}^{n}$ defined by

$$
f(\tau, \mathbf{y}):=\pi_{1}^{-} \tilde{\mathbf{x}}(\tau, \mathbf{y}), \quad \forall(\tau, \mathbf{y}) \in \mathbb{R} \times \operatorname{Fix}\left(R_{0}\right) .
$$

(In view of Lemma 9 we assume here for simplicity that the flow $\tilde{\mathbf{x}}(t, \mathbf{x}$ ) is globally defined for all $\left.(t, \mathbf{x}) \in \mathbb{R} \times \mathbb{R}^{n}\right)$. Our (local) continuation 
problem can then be described as follows: given a solution $(\tau, \mathbf{y})=$ $\left(T, \mathbf{y}_{0}\right) \in \mathbb{R} \times \operatorname{Fix}\left(R_{0}\right)$ (with $T>0$ ) of the equation

$$
f(\tau, \mathbf{y})=\mathbf{0},
$$

what can we say about the zero set of $f$ near $\left(T, \mathbf{y}_{0}\right)$ ?

Before we go on we should make the following observation. In the definition given by (21) we consider the mapping $f$ as taking its values in the space $\mathbb{R}^{n}$, but we could of course also consider it as a mapping taking values in the subspace $\operatorname{Fix}\left(-R_{1}\right)$ of $\mathbb{R}^{n}$. Actually, from the point of view of "submersivity properties" this last option seems to be the most advantageous one. However, in those cases where we have to take first integrals of (13) into account, we will have to rely on the general theory of Section 2 and this will only be possible by considering $f$ as taking values in $\mathbb{R}^{n}$. So, depending on the case under consideration, we will take the appropriate point of view.

The continuation problem is easiest to answer when the mapping $f: \mathbb{R} \times \operatorname{Fix}\left(R_{0}\right) \rightarrow \operatorname{Fix}\left(-R_{1}\right)$ is a submersion at some solution $\left(T, \mathbf{y}_{0}\right) \in$ $\mathbb{R} \times \operatorname{Fix}\left(R_{0}\right)$ of $(22)$, since then the zero set of $f$ will locally near $\left(T, \mathbf{y}_{0}\right)$ form a submanifold of dimension $1+n_{0}^{+}-n_{1}^{-}$. In the paper (Howison and Meyer, 2000) the corresponding doubly symmetric solution is called non-degenerate. In order to check the submersivity of $f$ we need to calculate the derivative of $f$ at $\left(T, \mathbf{y}_{0}\right)$; we find

$$
D f\left(T, \mathbf{y}_{0}\right) \cdot(\tau, \mathbf{y})=\pi_{1}^{-} M \mathbf{y}+\tau \pi_{1}^{-} X\left(\mathbf{z}_{0}\right)=\pi_{1}^{-} M \mathbf{y}+\tau X\left(\mathbf{z}_{0}\right),
$$

where $\mathbf{z}_{0}:=\tilde{\mathbf{x}}\left(T, \mathbf{y}_{0}\right) \in \operatorname{Fix}\left(R_{1}\right)$, and where

$$
M:=D_{\mathbf{x}} \tilde{\mathbf{x}}\left(T, \mathbf{y}_{0}\right) \in \mathcal{L}\left(\mathbb{R}^{n}\right) .
$$

To obtain the second equality in (23) we have used the fact that the "endpoint" $\mathbf{z}_{0}$ of the $\left(R_{0}, R_{1}\right)$-symmetric solution $\tilde{\mathbf{x}}\left(t, \mathbf{y}_{0}\right)$ belongs to $\operatorname{Fix}\left(R_{1}\right)$, and hence $X\left(\mathbf{z}_{0}\right) \in \operatorname{Fix}\left(-R_{1}\right)$. Introducing the solution $V$ : $\mathbb{R} \rightarrow \mathcal{L}\left(\mathbb{R}^{n}\right)$ of the initial value problem

$$
\dot{V}(t)=D X\left(\tilde{\mathbf{x}}\left(t, \mathbf{y}_{0}\right)\right) \cdot V(t), \quad V(0)=I,
$$

it is easily shown that $M=V(T)$. From $(23)$ and $\operatorname{Fix}\left(R_{0}\right)=\operatorname{Im} \pi_{0}^{+}$it follows that

$$
\begin{aligned}
\operatorname{Im}\left(D f\left(T, \mathbf{y}_{0}\right)\right) & =\pi_{1}^{-} M\left(\operatorname{Fix}\left(R_{0}\right)\right)+\mathbb{R} X\left(\mathbf{z}_{0}\right) \\
& =\operatorname{Im}\left(\pi_{1}^{-} M \pi_{0}^{+}\right)+\mathbb{R} X\left(\mathbf{z}_{0}\right) .
\end{aligned}
$$

In analogy with the case of periodic solutions (see Muñoz Almaraz et al. (2003)) and by lack of imagination to invent a more appropriate name we will call $M$ the monodromy matrix of the $\left(R_{0}, R_{1}\right)$ symmetric solution $\tilde{\mathbf{x}}\left(t, \mathbf{y}_{0}\right)$. The reader should be aware that in those 
cases where this solution is also periodic the matrix $M$ as defined here forms only a building block from which the real monodromy matrix can be reconstructed (using the symmetries).

The mapping $f: \mathbb{R} \times \operatorname{Fix}\left(R_{0}\right) \rightarrow \operatorname{Fix}\left(-R_{1}\right)$ is a submersion at $\left(T, \mathbf{y}_{0}\right)$ if and only if

$$
\operatorname{Im}\left(\pi_{1}^{-} M \pi_{0}^{+}\right)+\mathbb{R} X\left(\mathbf{z}_{0}\right)=\operatorname{Fix}\left(-R_{1}\right) .
$$

However, as we will explain now, this submersivity condition may fail due to the presence of some appropriate first integrals of (13).

We can write $f(\tau, \mathbf{y})=g(\tau, \mathbf{y})-h(\tau, \mathbf{y})$, with $g, h: \mathbb{R} \times \operatorname{Fix}\left(R_{0}\right) \rightarrow \mathbb{R}^{n}$ defined (for all $(\tau, \mathbf{y}) \in \mathbb{R} \times \operatorname{Fix}\left(R_{0}\right)$ ) by

$$
g(\tau, \mathbf{y}):=\tilde{\mathbf{x}}(\tau, \mathbf{y}) \text { and } h(\tau, \mathbf{y}):=\pi_{1}^{+} \tilde{\mathbf{x}}(\tau, \mathbf{y}) .
$$

(It is here that we are forced to consider $f$ as a mapping with values in $\left.\mathbb{R}^{n}\right)$. The mappings $g$ and $h$ satisfy the following "linear constraint":

$$
\pi_{1}^{+} g(\tau, \mathbf{y})=\pi_{1}^{+} h(\tau, \mathbf{y}), \quad \forall(\tau, \mathbf{y}) \in \mathbb{R} \times \operatorname{Fix}\left(R_{0}\right),
$$

but there may also be further constraints. Indeed, let $\mathscr{F}$ be the space of those $\mathscr{C}^{1}$-functions $F: \mathbb{R}^{n} \rightarrow \mathbb{R}$ which are a first integral of (13) (i.e. $D F(\mathbf{x}) \cdot X(\mathbf{x})=0$ for all $\mathbf{x} \in \mathbb{R}^{n}$ ), and which are constant on $\operatorname{Fix}\left(R_{0}\right) \cup \operatorname{Fix}\left(R_{1}\right)$. We have then for all $F \in \mathscr{F}$ and for all $(\tau, \mathbf{y}) \in$ $\mathbb{R} \times \operatorname{Fix}\left(R_{0}\right)$ that

$$
F(g(\tau, \mathbf{y}))=F(\tilde{\mathbf{x}}(\tau, \mathbf{y}))=F(\mathbf{y})=F(\mathbf{0})=F\left(\pi_{1}^{+} \tilde{\mathbf{x}}(\tau, \mathbf{y})\right)=F(h(\tau, \mathbf{y})) .
$$

Combining this with (28), (25) and Lemma 3 we obtain the following.

LEMMA 12. Let $\left(T, \mathbf{y}_{0}\right) \in \mathbb{R} \times \operatorname{Fix}\left(R_{0}\right)$ (with $T>0$ ) be a solution of (22), and let $\mathbf{z}_{0}:=\tilde{\mathbf{x}}\left(T, \mathbf{y}_{0}\right)$. Then

$$
\begin{aligned}
& \operatorname{Im}\left(D f\left(T, \mathbf{y}_{0}\right)\right) \\
& =\operatorname{Im}\left(\pi_{1}^{-} M \pi_{0}^{+}\right)+\mathbb{R} X\left(\mathbf{z}_{0}\right) \subset \bigcap_{F \in \mathscr{F}} \operatorname{Ker}\left(D F\left(\mathbf{z}_{0}\right)\right) \cap \operatorname{Ker}\left(\pi_{1}^{+}\right) .
\end{aligned}
$$

This result leads in a natural way to the following definition.

DEFINITION 13. Under the conditions of Lemma 12 we say that the $\left(R_{0}, R_{1}\right)$-symmetric solution $\tilde{\mathbf{x}}\left(t, \mathbf{y}_{0}\right)$ is normal if we have equality in $(29)$, i.e. if

$$
\operatorname{Im}\left(\pi_{1}^{-} M \pi_{0}^{+}\right)+\mathbb{R} X\left(\mathbf{z}_{0}\right)=\bigcap_{F \in \mathscr{F}} \operatorname{Ker}\left(D F\left(\mathbf{z}_{0}\right)\right) \cap \operatorname{Ker}\left(\pi_{1}^{+}\right) .
$$


Clearly this means that $\left(T, \mathbf{y}_{0}\right)$ is a normal zero of the constrained mapping $f$ defined by (21). (To be precise: $f$ has to be considered as a mapping from $\mathbb{R} \times \operatorname{Fix}\left(R_{0}\right)$ into $\mathbb{R}^{n}$, and the constraints are given by $F \in \mathscr{F}$ and by $\pi_{1}^{+}$). This definition of a normal doubly symmetric solution is analogous to that of a normal periodic solution as given in (Muñoz Almaraz et al., 2003) (see also subsection 2.3). From Theorem 5 and Corollary 7 we obtain directly the following main result on the continuation of normal doubly symmetric solutions.

THEOREM 14. Let $\mathbf{y}_{0} \in \operatorname{Fix}\left(R_{0}\right)$ be such that $\tilde{\mathbf{x}}\left(t, \mathbf{y}_{0}\right)$ is a normal $\left(R_{0}, R_{1}\right)$-symmetric solution of (13), with basic domain $[0, T]$; let $\mathbf{z}_{0}:=$ $\tilde{\mathbf{x}}\left(T, \mathbf{y}_{0}\right)$,

$$
\mathscr{W}:=\left\{D F\left(\mathbf{z}_{0}\right) \mid F \in \mathscr{F}\right\} \subset \mathcal{L}\left(\mathbb{R}^{n} ; \mathbb{R}\right),
$$

and $k:=\operatorname{dim} \mathscr{W}$. Then $\tilde{\mathbf{x}}\left(t, \mathbf{y}_{0}\right)$ belongs locally to a m-parameter family of normal $\left(R_{0}, R_{1}\right)$-symmetric solutions, with $m=1+n_{0}^{+}-n_{1}^{-}+k$

Proof. Under the assumptions of the theorem $\left(T, \mathbf{y}_{0}\right)$ is a normal zero of the mapping $f$, and the mapping $f$ is a quasi-submersion at $\left(T, \mathbf{y}_{0}\right)$. The theorem then follows from the general results of Section 2, with the dimension $m$ of the family given by $m=\operatorname{dim} \operatorname{Ker}\left(D f\left(T, \mathbf{y}_{0}\right)\right)=$ $1+n_{0}^{+}-\operatorname{dim} \operatorname{Im}\left(D f\left(T, \mathbf{y}_{0}\right)\right)$. In order to determine $\operatorname{dim} \operatorname{Ker}\left(D f\left(T, \mathbf{y}_{0}\right)\right)$ consider the space $\mathscr{W}$ defined in the statement, and let

$$
\mathscr{W}^{\perp}:=\bigcap_{F \in \mathscr{F}} \operatorname{Ker}\left(D F\left(\mathbf{z}_{0}\right)\right) \subset \mathbb{R}^{n} .
$$

Clearly $\operatorname{dim} \mathscr{W}^{\perp}=n-\operatorname{dim} \mathscr{W}=n-k$. Using the facts that each $F \in \mathscr{F}$ is constant on $\operatorname{Fix}\left(R_{1}\right)=\operatorname{Im}\left(\pi_{1}^{+}\right)$and that $\mathbb{R}^{n}=\operatorname{Im}\left(\pi_{1}^{+}\right) \oplus \operatorname{Ker}\left(\pi_{1}^{+}\right)$it follows easily that

$$
\operatorname{Im}\left(\pi_{1}^{+}\right) \subset \mathscr{W}^{\perp} \quad \text { and } \quad \mathscr{W}^{\perp}=\left(\mathscr{W}^{\perp} \cap \operatorname{Ker}\left(\pi_{1}^{+}\right)\right) \oplus \operatorname{Im}\left(\pi_{1}^{+}\right) .
$$

Since $\tilde{\mathbf{x}}\left(t, \mathbf{y}_{0}\right)$ is a normal doubly periodic solution we have from (25) and (30) that $\operatorname{Im}\left(D f\left(T, \mathbf{y}_{0}\right)\right)=\mathscr{W}^{\perp} \cap \operatorname{Ker}\left(\pi_{1}^{+}\right)$, and hence

$$
\operatorname{dim} \operatorname{Im}\left(D f\left(T, \mathbf{y}_{0}\right)\right)=\operatorname{dim} \mathscr{W}^{\perp}-n_{1}^{+}=n-k-n_{1}^{+}=n_{1}^{-}-k .
$$

Bringing this in the earlier expression for $m$ we find $m=1+n_{0}^{+}-$ $\left(n_{1}^{-}-k\right)=1+n_{0}^{+}-n_{1}^{-}+k$, which proves the theorem.

Some special cases appear when $n_{0}^{+}=n_{1}^{-}$; then $m=1+k$, and if moreover $k=0$ (no nontrivial first integrals which are constant on $\left.\operatorname{Fix}\left(R_{0}\right) \cup \operatorname{Fix}\left(R_{1}\right)\right)$ then $m=1$. This is for example the case with the non-degenerate doubly symmetric solutions studied in Howison and Meyer (2000). 
The following heuristic argument may give some better insight in the continuation result of Theorem 14. Under the assumptions of the theorem let $F_{j} \in \mathscr{F}(1 \leq j \leq k)$ be such that $\left\{D F_{j}\left(\mathbf{z}_{0}\right) \mid 1 \leq j \leq k\right\}$ forms a basis of $\mathscr{W}$, and let $f_{j}:=F_{j}\left(\mathbf{y}_{0}\right)=F_{j}\left(\mathbf{z}_{0}\right)$. Then the "level set" $\mathscr{M}_{0}:=\left\{\mathbf{x} \in \mathbb{R}^{n} \mid F_{j}(\mathbf{x})=f_{j}, 1 \leq j \leq k\right\}$ is (at least locally) a submanifold of dimension $(n-k)$ which contains next to the orbit $\left\{\tilde{\mathbf{x}}\left(t, \mathbf{y}_{0}\right) \mid t \in \mathbb{R}\right\}$ also the subspaces $\operatorname{Fix}\left(R_{0}\right)$ and $\operatorname{Fix}\left(R_{1}\right)$ (this is where we use the definition of $\mathscr{F}$ as the space of all first integrals which are constant on $\left.\operatorname{Fix}\left(R_{0}\right) \cup \operatorname{Fix}\left(R_{1}\right)\right)$. The fact that $f\left(T, \mathbf{y}_{0}\right)=\mathbf{0}$ means that $\mathbf{z}_{0} \in \tilde{\mathbf{x}}\left(T, \operatorname{Fix}\left(R_{0}\right)\right) \cap \operatorname{Fix}\left(R_{1}\right) \cap \mathscr{M}_{0}$. Typically we should have at $\mathbf{z}_{0}$ a transversal intersection of the $n_{0}^{+}$-dimensional manifold $\tilde{\mathbf{x}}\left(T, \operatorname{Fix}\left(R_{0}\right)\right)$ with the $n_{1}^{+}$-dimensional manifold $\operatorname{Fix}\left(R_{1}\right)$ within the $(n-k)$-dimensional manifold $\mathscr{M}_{0}$; this transversality forms the geometric interpretation of the most typical (although not the most general) case of the normality condition. If such transversality is satisfied then the set $\tilde{\mathbf{x}}\left(T, \operatorname{Fix}\left(R_{0}\right)\right) \cap \operatorname{Fix}\left(R_{1}\right) \cap \mathscr{M}_{0}$ will locally be a submanifold of dimension $n_{0}^{+}+n_{1}^{+}-(n-k)=n_{0}^{+}-n_{1}^{-}+k$; each of the points in this intersection generates a $\left(R_{0}, R_{1}\right)$-symmetric solution. Moreover, for each $\tau \in \mathbb{R}$ near $T$ also the intersection $\tilde{\mathbf{x}}\left(\tau, \operatorname{Fix}\left(R_{0}\right)\right) \cap \operatorname{Fix}\left(R_{1}\right)$ will be transversal in $\mathscr{M}_{0}$, leading to a $\left(n_{0}^{+}-n_{1}^{-}+k\right)$-parameter family of $\left(R_{0}, R_{1}\right)$-symmetric solutions, this time with basic domain $[0, \tau]$. Taken together all these intersection points form the $m$-dimensional manifold given by Theorem 14. In subsection 3.4 we will clarify this further in the particular case of Hamiltonian systems.

From Theorem 14 we also get an alternative criterion for the normality of a doubly symmetric solution; in the formulation we use the following subspace of $\operatorname{Fix}\left(R_{0}\right)$ :

$$
\mathcal{T}:=\left\{\mathbf{y} \in \operatorname{Fix}\left(R_{0}\right) \mid \exists \alpha \in \mathbb{R}: \pi_{1}^{-} M \mathbf{y}=\alpha X\left(\mathbf{z}_{0}\right)\right\} .
$$

COROLLARY 15. A $\left(R_{0}, R_{1}\right)$-symmetric solution $\tilde{\mathbf{x}}\left(t, \mathbf{y}_{0}\right)$ with basic domain $[0, T]$ and endpoint $\mathbf{z}_{0}=\tilde{\mathbf{x}}\left(T, \mathbf{y}_{0}\right)$ is normal if and only if

$$
\operatorname{dim} \mathcal{T}=1+n_{0}^{+}-n_{1}^{-}+k,
$$

with $k=\operatorname{dim} \mathscr{W}$ as in Theorem 14. When the condition (34) is satisfied then $\mathcal{T}$ is the tangent space at $\mathbf{y}_{0}$ to the set of initial points in $\operatorname{Fix}\left(R_{0}\right)$ which generate a $\left(R_{0}, R_{1}\right)$-symmetric solution.

Proof. It follows from the inclusion (29) and from the proof of Theorem 14 that $\tilde{\mathbf{x}}\left(t, \mathbf{y}_{0}\right)$ will be a normal $\left(R_{0}, R_{1}\right)$-symmetric solution if and only if (32) holds, that is, if and only if $\operatorname{dim} \operatorname{Ker}\left(D f\left(T, \mathbf{y}_{0}\right)\right)=$ $1+n_{0}^{+}-n_{1}^{-}+k$. Also, when this normality condition is satisfied $\operatorname{Ker}\left(D f\left(T, \mathbf{y}_{0}\right)\right)$ forms the tangent space at $\left(T, \mathbf{y}_{0}\right)$ to the zero set $\left\{(\tau, \mathbf{y}) \in \mathbb{R} \times \operatorname{Fix}\left(R_{0}\right) \mid f(\tau, \mathbf{y})=\mathbf{0}\right\}$ of $f$. From (23) we see that

$$
\operatorname{Ker}\left(D f\left(T, \mathbf{y}_{0}\right)\right)=\left\{(\tau, \mathbf{y}) \in \mathbb{R} \times \operatorname{Fix}\left(R_{0}\right) \mid \pi_{1}^{-} M \mathbf{y}+\tau X\left(\mathbf{z}_{0}\right)=\mathbf{0}\right\},
$$


and hence $\mathcal{T}$ is the projection on $\operatorname{Fix}\left(R_{0}\right)$ of $\operatorname{Ker}\left(D f\left(T, \mathbf{y}_{0}\right)\right)$. One can directly verify that $\operatorname{Ker}\left(D f\left(T, \mathbf{y}_{0}\right)\right)$ contains no nontrivial elements of the form $(\tau, \mathbf{0})$, from which it follows that $\operatorname{dim} \mathcal{T}=\operatorname{dim} \operatorname{Ker}\left(D f\left(T, \mathbf{y}_{0}\right)\right)$ and that $\mathcal{T}$ is the tangent space at $\mathbf{y}_{0}$ of the projection of the zero set of $f$ on the space $\operatorname{Fix}\left(R_{0}\right)$. This proves the corollary.

In the special case where $R_{1}=R_{0}$ the equation (22) takes the form

$$
f_{0}(\tau, \mathbf{y}):=\pi_{0}^{-} \tilde{\mathbf{x}}(\tau, \mathbf{y})=\mathbf{0} .
$$

The $R_{0}$-symmetric periodic solution $\tilde{\mathbf{x}}\left(t, \mathbf{y}_{0}\right)$ generated by a solution $\left(T, \mathbf{y}_{0}\right) \in \mathbb{R} \times \operatorname{Fix}\left(R_{0}\right)(T>0)$ of (35) will be normal if

$$
\operatorname{Im}\left(\pi_{0}^{-} M \pi_{0}^{+}\right)+\mathbb{R} X\left(\mathbf{z}_{0}\right)=\bigcap_{F \in \mathscr{F}} \operatorname{Ker}\left(D F\left(\mathbf{z}_{0}\right)\right) \cap \operatorname{Ker}\left(\pi_{0}^{+}\right) ;
$$

as before $\mathbf{z}_{0}=\tilde{\mathbf{x}}\left(T, \mathbf{y}_{0}\right) \in \operatorname{Fix}\left(R_{0}\right)$ is the endpoint of the solution. From Theorem 14 and Corollary 15 we obtain then the following.

THEOREM 16. Let $\left(T, \mathbf{y}_{0}\right) \in \mathbb{R} \times \operatorname{Fix}\left(R_{0}\right)$ be a solution of (35) such that (36) is satisfied, and let $\mathbf{z}_{0}=\tilde{\mathbf{x}}\left(T, \mathbf{y}_{0}\right)$. Then $\tilde{\mathbf{x}}\left(t, \mathbf{y}_{0}\right)$ is a normal $R_{0}$-symmetric periodic orbit, belonging to a $m=\left(1+n_{0}^{+}-n_{0}^{-}+k\right)$ parameter family of such normal $R_{0}$-symmetric periodic orbits; also,

$$
\mathcal{T}_{0}:=\left\{\mathbf{y} \in \operatorname{Fix}\left(R_{0}\right) \mid \exists \alpha \in \mathbb{R}: \pi_{0}^{-} M \mathbf{y}=\alpha X\left(\mathbf{z}_{0}\right)\right\}
$$

forms the tangent space at $\mathbf{y}_{0}$ to the manifold of initial points in $\operatorname{Fix}\left(R_{0}\right)$ of this family.

To conclude this subsection we observe that from Corollary 11 we know that a doubly symmetric solution with basic domain $[0, T]$ is also doubly symmetric with basic domain $[\ell T,(\ell+1) T]$, for any $\ell \in \mathbb{Z}$ and with appropriately adjusted symmetry operators. Using Corollary 15 one can prove the following result which essentialy says that normality is an inherent property of a doubly symmetric solution, independent of the chosen basic domain; we omit the proof.

PROPOSITION 17. Let $\hat{\mathbf{x}}: \mathbb{R} \rightarrow \mathbb{R}^{n}$ be a normal $\left(R_{0}, R_{1}\right)$-symmetric solution of (13), with basic domain $[0, T]$, and let $\ell \in \mathbb{Z}$. Then $\hat{\mathbf{x}}$ is also normal when considered as a $\left(\left(R_{1} R_{0}\right)^{\ell-1} R_{1},\left(R_{1} R_{0}\right)^{\ell} R_{1}\right)$-symmetric solution with basic domain $[\ell T,(\ell+1) T]$. 


\subsection{AN ASSOCIATED BOUNDARY VALUE PROBLEM}

In this subsection we describe a method for obtaining the "continuation manifold" given by Theorem 14 (or Theorem 16) via regular continuation, that is, by directly using the implicit function theorem. The general theory of Section 2 and in particular Corollary 6 show us how this can be done, but here we will present an equivalent but slightly different approach which is better suited for numerical treatment using BVP-solvers such as AUTO (Doedel et al, 2000). The arguments which we will use are similar to those in (Muñoz Almaraz et al., 2003). We choose any inner product $\langle\cdot, \cdot\rangle$ on $\mathbb{R}^{n}$ to define the gradient $\nabla F: \mathbb{R}^{n} \rightarrow$ $\mathbb{R}^{n}$ of a smooth function $F: \mathbb{R}^{n} \rightarrow \mathbb{R}$.

LEMMA 18. Let $F \in \mathscr{F}$, and let $\hat{\mathbf{x}}: J \subset \mathbb{R} \rightarrow \mathbb{R}^{n}$ be a solution of

$$
\dot{\mathbf{x}}=X(\mathbf{x})+\nabla F(\mathbf{x})
$$

such that for some $t_{0}, t_{1} \in J\left(t_{1}>t_{0}\right)$ we have $\hat{\mathbf{x}}\left(t_{0}\right) \in \operatorname{Fix}\left(R_{0}\right)$ and $\hat{\mathbf{x}}\left(t_{1}\right) \in \operatorname{Fix}\left(R_{1}\right)$. Then $\hat{\mathbf{x}}$ is on $\left[t_{0}, t_{1}\right]$ actually a solution of (13).

Proof. Since $F$ is constant on $\operatorname{Fix}\left(R_{0}\right) \cup \operatorname{Fix}\left(R_{1}\right)$ and a first integral of the vector field $X(\mathbf{x})$ we have that

$$
\begin{aligned}
0 & =F\left(\hat{\mathbf{x}}\left(t_{1}\right)\right)-F\left(\hat{\mathbf{x}}\left(t_{0}\right)\right)=\int_{t_{0}}^{t_{1}} D F(\hat{\mathbf{x}}(t)) \cdot(X(\hat{\mathbf{x}}(t))+\nabla F(\hat{\mathbf{x}}(t))) d t \\
& =\int_{t_{0}}^{t_{1}}\langle\nabla F(\hat{\mathbf{x}}(t)), \nabla F(\hat{\mathbf{x}}(t))\rangle d t .
\end{aligned}
$$

Hence $\nabla F(\hat{\mathbf{x}}(t))=\mathbf{0}$ for all $t \in\left[t_{0}, t_{1}\right]$, which proves the lemma.

Now suppose that $\tilde{\mathbf{x}}\left(t, \mathbf{y}_{0}\right)$ is a $\left(R_{0}, R_{1}\right)$-symmetric solution of (13), with basic domain $[0, T]$ and endpoint $\mathbf{z}_{0}=\tilde{\mathbf{x}}\left(T, \mathbf{y}_{0}\right)$. As in the discussion after Theorem 14 we let $F_{j} \in \mathscr{F}(1 \leq j \leq k)$ be such that $\left\{D F_{j}\left(\mathbf{z}_{0}\right) \mid 1 \leq j \leq k\right\}$ forms a basis of $\mathscr{W}=\left\{D F\left(\mathbf{z}_{0}\right) \mid F \in \mathscr{F}\right\}$. Instead of modifying the equation (22) as suggested by the results of Section 2 we will modify the differential equation (13). Namely, we consider the modified system

$$
\dot{\mathbf{x}}=X(\mathbf{x})+\sum_{j=1}^{k} \alpha_{j} \nabla F_{j}(\mathbf{x}),
$$

thus introducing $k$ new parameters $\alpha_{j} \in \mathbb{R}(1 \leq j \leq k)$. We denote the flow of (38) by $\tilde{\mathbf{x}}_{\text {mod }}(t, \mathbf{x}, \alpha)$, with $\alpha=\left(\alpha_{1}, \alpha_{2}, \ldots, \alpha_{k}\right) \in \mathbb{R}^{k}$; clearly $\tilde{\mathbf{x}}_{\text {mod }}(t, \mathbf{x}, 0)=\tilde{\mathbf{x}}(t, \mathbf{x})$. We also define a mapping $\phi: \mathbb{R} \times \operatorname{Fix}\left(R_{0}\right) \times \mathbb{R}^{k} \rightarrow$ $\operatorname{Fix}\left(-R_{1}\right)$ by setting $\phi(\tau, \mathbf{y}, \alpha):=\pi_{1}^{-} \tilde{\mathbf{x}}_{\text {mod }}(\tau, \mathbf{y}, \alpha)$ for all $(\tau, \mathbf{y}, \alpha) \in$ $\mathbb{R} \times \operatorname{Fix}\left(R_{0}\right) \times \mathbb{R}^{k}$; again, $\phi(\tau, \mathbf{y}, 0)=f(\tau, \mathbf{y})$. Now we can state the main result of this subsection. 
THEOREM 19. Let $\tilde{\mathbf{x}}\left(t, \mathbf{y}_{0}\right)$ be a normal $\left(R_{0}, R_{1}\right)$-symmetric solution of (13), with basic domain $[0, T]$ and endpoint $\mathbf{z}_{0}$. Define $\mathscr{W}, k$ and $\phi: \mathbb{R} \times \operatorname{Fix}\left(R_{0}\right) \times \mathbb{R}^{k} \rightarrow \operatorname{Fix}\left(-R_{1}\right)$ as in the preceding paragraph. Then $\phi\left(T, \mathbf{y}_{0}, 0\right)=\mathbf{0}$, the mapping $\phi$ is a submersion at $\left(T, \mathbf{y}_{0}, 0\right)$, the zero set of $\phi$ is locally a $m=\left(1+n_{0}^{+}-n_{1}^{-}+k\right)$-dimensional manifold along which $\alpha \equiv 0$, and the projection of this set on $\operatorname{Fix}\left(R_{0}\right)$ forms a m-dimensional manifold of initial points which generate normal $\left(R_{0}, R_{1}\right)$-symmetric solutions of (13) with basic domain $[0, \tau], \tau$ close to $T$.

Proof. It is obvious that $\phi\left(T, \mathbf{y}_{0}, 0\right)=\mathbf{0}$. To show that $\phi$ is a submersion at $\left(T, \mathbf{y}_{0}, 0\right)$ we start by calculating $D \phi\left(T, \mathbf{y}_{0}, 0\right)$. Using the fact that $\phi(\tau, \mathbf{y}, 0)=f(\tau, \mathbf{y})$ and introducing

$$
\psi_{j}(t):=\frac{\partial \tilde{\mathbf{x}}_{m o d}}{\partial \alpha_{j}}\left(t, \mathbf{y}_{0}, 0\right) \quad \text { and } \quad \mathbf{w}_{j}:=\psi_{j}(T), \quad(1 \leq j \leq k),
$$

we find that

$$
D \phi\left(T, \mathbf{y}_{0}, 0\right) \cdot(\tau, \mathbf{y}, \alpha)=D f\left(T, \mathbf{y}_{0}\right) \cdot(\tau, \mathbf{y})+\sum_{j=1}^{k} \alpha_{j} \pi_{1}^{-} \mathbf{w}_{j} .
$$

Since $\psi_{j}(t)$ satisfies the initial value problem

$$
\dot{\psi}_{j}(t)=D X\left(\tilde{\mathbf{x}}\left(t, \mathbf{y}_{0}\right)\right) \psi_{j}(t)+\nabla F_{j}\left(\tilde{\mathbf{x}}\left(t, \mathbf{y}_{0}\right)\right), \quad \psi_{j}(0)=\mathbf{0},
$$

we can use the transition matrix $V(t)$ introduced earlier in this section to rewrite $\psi_{j}(t)$ in the form $\psi_{j}(t)=V(t) \int_{0}^{t} V(s)^{-1} \nabla F_{j}\left(\tilde{\mathbf{x}}\left(s, \mathbf{y}_{0}\right)\right) d s$. Hence

$$
\mathbf{w}_{j}=V(T) \int_{0}^{T} V(s)^{-1} \nabla F_{j}\left(\tilde{\mathbf{x}}\left(s, \mathbf{y}_{0}\right)\right) d s, \quad(1 \leq j \leq k) .
$$

To prove that $D \phi\left(T, \mathbf{y}_{0}, 0\right) \in \mathcal{L}\left(\mathbb{R} \times \operatorname{Fix}\left(R_{0}\right) \times \mathbb{R}^{k} ; \operatorname{Fix}\left(-R_{1}\right)\right)$ is surjective (and $\phi$ a submersion) it is sufficient to show that

$$
\operatorname{Ker} D \phi\left(T, \mathbf{y}_{0}, 0\right)=\operatorname{Ker} D f\left(T, \mathbf{y}_{0}\right) \times\{0\} ;
$$

indeed, it follows from the normality of $\tilde{\mathbf{x}}\left(t, \mathbf{y}_{0}\right)$ and our earlier calculations that $\operatorname{dim} \operatorname{Ker}\left(D f\left(T, \mathbf{y}_{0}\right)\right)=1+n_{0}^{+}-n_{1}^{-}+k$, which in combination with (40) proves that

$$
\begin{aligned}
& \operatorname{dim} \operatorname{Ker}\left(D \phi\left(T, \mathbf{y}_{0}, 0\right)\right)+\operatorname{dim} \operatorname{Fix}\left(-R_{1}\right) \\
& \quad=1+n_{0}^{+}+k=\operatorname{dim}\left(\mathbb{R} \times \operatorname{Fix}\left(R_{0}\right) \times \mathbb{R}^{k}\right) .
\end{aligned}
$$

So let $(\tau, \mathbf{y}, \alpha) \in \mathbb{R} \times \operatorname{Fix}\left(R_{0}\right) \times \mathbb{R}^{k}$ be such that

$$
D f\left(T, \mathbf{y}_{0}\right) \cdot(\tau, \mathbf{y})+\sum_{j=1}^{k} \alpha_{j} \pi_{1}^{-} \mathbf{w}_{j}=\mathbf{0}
$$


We set $F:=\sum_{j=1}^{k} \alpha_{j} F_{j} \in \mathscr{F}$ and apply $D F\left(\mathbf{z}_{0}\right)$ to the foregoing equality. From (29) and the fact that $F$ is constant on $\operatorname{Fix}\left(R_{1}\right)$ (which implies that $\left.D F\left(\mathbf{z}_{0}\right) \pi_{1}^{-}=D F\left(\mathbf{z}_{0}\right)\right)$ we obtain $\sum_{j=1}^{k} \alpha_{j} D F\left(\mathbf{z}_{0}\right) \cdot \mathbf{w}_{j}=\mathbf{0}$, or more explicitly using (39):

$$
\int_{0}^{T} D F\left(\tilde{\mathbf{x}}\left(T, \mathbf{y}_{0}\right)\right) V(T) V(s)^{-1} \nabla F\left(\tilde{\mathbf{x}}\left(s, \mathbf{y}_{0}\right)\right) d s=\mathbf{0} .
$$

Differentiating the identity $F(\tilde{\mathbf{x}}(t, \mathbf{x}))=F(\mathbf{x})$ at $\mathbf{x}=\mathbf{y}_{0}$ shows that

$$
D F\left(\tilde{\mathbf{x}}\left(t, \mathbf{y}_{0}\right)\right) V(t)=D F\left(\mathbf{y}_{0}\right), \quad \forall t \in \mathbb{R} ;
$$

so we can replace $D F\left(\tilde{\mathbf{x}}\left(T, \mathbf{y}_{0}\right)\right) V(T)$ by $D F\left(\tilde{\mathbf{x}}\left(s, \mathbf{y}_{0}\right)\right) V(s)$ in the foregoing integral, giving us

$$
\int_{0}^{T}\left\langle\nabla F\left(\tilde{\mathbf{x}}\left(s, \mathbf{y}_{0}\right)\right), \nabla F\left(\tilde{\mathbf{x}}\left(s, \mathbf{y}_{0}\right)\right)\right\rangle d s=\mathbf{0} .
$$

We conclude that $\nabla F\left(\tilde{\mathbf{x}}\left(s, \mathbf{y}_{0}\right)\right)=\mathbf{0}$ for all $s \in[0, T]$, and in particular for $s=T$; therefore $\sum_{j=1}^{k} \alpha_{j} \nabla F_{j}\left(\mathbf{z}_{0}\right)=\mathbf{0}$ and $\alpha_{j}=0(1 \leq j \leq k)$, since the vectors $\nabla F_{j}\left(\mathbf{z}_{0}\right)(1 \leq j \leq k)$ are linearly independent. This proves (40).

The remaining part of the theorem follows rather easily. The zero set of $\phi$ can locally near $\left(T, \mathbf{y}_{0}, 0\right)$ be obtained by using the implicit function theorem and forms a submanifold with dimension equal to $\operatorname{dim} \operatorname{Ker}\left(D \phi\left(T, \mathbf{y}_{0}, 0\right)\right)=\operatorname{dim} \operatorname{Ker}\left(D f\left(T, \mathbf{y}_{0}\right)\right)=1+n_{0}^{+}-n_{1}^{-}+k$. If $(\tau, \mathbf{y}, \alpha)$ is such a zero of $\phi$ then Lemma 18 implies that

$$
\sum_{j=1}^{k} \alpha_{j} \nabla F_{j}\left(\tilde{\mathbf{x}}_{\text {mod }}(t, \mathbf{y}, \alpha)\right)=\mathbf{0}
$$

for all $t \in[0, \tau]$, and in particular for $t=\tau$; since $\tilde{\mathbf{x}}_{\text {mod }}(\tau, \mathbf{y}, \alpha)$ is close to $\mathbf{z}_{0}=\tilde{\mathbf{x}}_{\text {mod }}\left(T, \mathbf{y}_{0}, 0\right)$ the vectors $\nabla F_{j}\left(\tilde{\mathbf{x}}_{\text {mod }}(\tau, \mathbf{y}, \alpha)\right)(1 \leq j \leq k)$ will be linearly independent, and we conclude again that $\alpha_{j}=0$ for $1 \leq j \leq k$ and hence $f(\tau, \mathbf{y})=\mathbf{0}$. The remaining statements then follow from our earlier results.

In order to implement Theorem 19 one can apply a time rescale to (38) such as to transform the (a priori unknown) basic domain $[0, \tau]$ into the fixed interval $[0,1]$, but with $\tau$ appearing as a parameter in the equations. This leads to the following boundary value problem for doubly symmetric solutions.

$\left(\right.$ DS-BVP) Find a $\mathscr{C}^{1}$-mapping $\mathbf{x}:[0,1] \rightarrow \mathbb{R}^{n}$ such that

$$
\left\{\begin{array}{l}
\dot{\mathbf{x}}=\tau\left(X(\mathbf{x})+\sum_{j=1}^{k} \alpha_{j} \nabla F_{j}(\mathbf{x})\right), \\
\mathbf{x}(0) \in \operatorname{Fix}\left(R_{0}\right) \\
\mathbf{x}(1) \in \operatorname{Fix}\left(R_{1}\right) .
\end{array}\right.
$$


In the theoretical setup as explained in this section one must start the continuation from a given normal $\left(R_{0}, R_{1}\right)$-symmetric solution, say with startpoint $\mathbf{y}_{0} \in \operatorname{Fix}\left(R_{0}\right)$, endpoint $\mathbf{z}_{0} \in \operatorname{Fix}\left(R_{1}\right)$ and basic domain $[0, T]$. The first integrals $F_{j} \in \mathscr{F}$ appearing in (41) must then be such that $\left\{\nabla F_{j}\left(\mathbf{z}_{0}\right) \mid 1 \leq j \leq k\right\}$ forms a basis of $\left\{\nabla F\left(\mathbf{z}_{0}\right) \mid F \in \mathscr{F}\right\}$. For all nearby solutions of (41) we will then have that $\alpha_{1}=\alpha_{2}=\cdots=$ $\alpha_{k}=0$, and therefore they correspond to (normal $\left(R_{0}, R_{1}\right)$-symmetric) solutions of (13). In a practical setup one can make a guess about which first integrals $F_{j}$ should appear in (41) and then try to obtain numerically some solution manifolds of (41).

We make a few final remarks before considering the Hamiltonian case. First, the results of this section can be easily extended to the case where the vectorfield $X$ depends on some external parameters. Second, according to the theory the solution manifolds of (41) will have dimension $m=1+n_{0}^{+}-n_{1}^{-}+k$, or $m=1+k$ in the frequently appearing case that $n_{0}^{+}=n_{0}^{-}=n_{1}^{+}=n_{1}^{-}$. In order to use certain numerical continuation schemes which allow only 1-dimensional solution branches (such as for example the pseudo-arclength method, see (Keller, 1977)) one has to add to the boundary value problem (41) $k$ appropriate "phase conditions". In the Hamiltonian case where first integrals are related to symmetries this can be done in a similar way as in (Muñoz Almaraz et al., 2003); see also subsection 3.4 and the examples in Section 4.

\subsection{Doubly Symmetric SOlutions in Hamiltonian Systems}

In this subsection we consider the particular case of Hamiltonian systems; more details on reversors in Hamiltonian systems can be found in e.g. (Meyer, 1981). In its simplest form the setting for this case can be described as follows. We take $n=2 N$, use on the phase space $\mathbb{R}^{2 N}$ the standard inner product $\langle\mathbf{x}, \mathbf{y}\rangle:=\sum_{j=1}^{2 N} x_{j} y_{j}$, and introduce the symplectic form $\omega: \mathbb{R}^{2 N} \times \mathbb{R}^{2 N} \rightarrow \mathbb{R}$ by setting $\omega(\mathbf{x}, \mathbf{y}):=\langle\mathbf{x}, J \mathbf{y}\rangle$, where $J \in \mathcal{L}\left(\mathbb{R}^{2 N}\right)$ is the standard symplectic matrix given by

$$
J:=\left[\begin{array}{cc}
O_{N} & I_{N} \\
-I_{N} & O_{N}
\end{array}\right]
$$

To each sufficiently smooth function $H: \mathbb{R}^{2 N} \rightarrow \mathbb{R}$ we associate the Hamiltonian vectorfield $X_{H}: \mathbb{R}^{2 N} \rightarrow \mathbb{R}^{2 N}$ defined by the relation

$$
D H(\mathbf{x}) \cdot \tilde{\mathbf{x}}=\omega\left(X_{H}(\mathbf{x}), \tilde{\mathbf{x}}\right), \quad \forall \mathbf{x}, \tilde{\mathbf{x}} \in \mathbb{R}^{2 n} .
$$

Explicitly we have $X_{H}(\mathbf{x})=J \nabla H(\mathbf{x})$. A $\mathscr{C}^{1}$-function $F: \mathbb{R}^{2 N} \rightarrow \mathbb{R}$ is a first integral of the Hamiltonian system

$$
\dot{\mathbf{x}}=X_{H}(\mathbf{x})
$$


if and only if $\{H, F\}(\mathbf{x})=0$ for all $\mathbf{x} \in \mathbb{R}^{2 N}$, where the Poisson bracket $\{H, F\}: \mathbb{R}^{2 N} \rightarrow \mathbb{R}$ is defined by $\{H, F\}(\mathbf{x}):=\omega\left(X_{H}(\mathbf{x}), X_{F}(\mathbf{x})\right)$. By the anti-symmetry of $\omega$ and the Poisson bracket it follows that (i) $H$ is a first integral of (44), and (ii) $F$ is a first integral of (44) if and only if $H$ is a first integral of the Hamiltonian system $\dot{\mathbf{x}}=X_{F}(\mathbf{x})$. This in turn implies that the flow $\tilde{\mathbf{x}}_{H}$ of (44) commutes with the flow $\tilde{\mathbf{x}}_{F}$ of $\dot{\mathbf{x}}=X_{F}(\mathbf{x})$, i.e.

$$
\tilde{\mathbf{x}}_{H}\left(t, \tilde{\mathbf{x}}_{F}(s, \mathbf{x})\right)=\tilde{\mathbf{x}}_{F}\left(s, \tilde{\mathbf{x}}_{H}(t, \mathbf{x})\right), \quad \forall t, s \in \mathbb{R}, \forall \mathbf{x} \in \mathbb{R}^{2 N} .
$$

Modulo some minor technical conditions also the converse is true: (45) implies that $F$ is a first integral of (44) - this is the Hamiltonian version of the celebrated Noether's Theorem (see e.g. (Meyer, 1999)).

A linear operator $R \in \mathcal{L}\left(\mathbb{R}^{2 N}\right)$ is symplectic if $\omega(R \mathbf{x}, R \mathbf{y})=\omega(\mathbf{x}, \mathbf{y})$ for all $\mathbf{x}, \mathbf{y} \in \mathbb{R}^{2 N}$, and anti-symplectic if $\omega(R \mathbf{x}, R \mathbf{y})=-\omega(\mathbf{x}, \mathbf{y})$ for all $\mathbf{x}, \mathbf{y} \in \mathbb{R}^{2 N}$; these conditions are equivalent to respectively $R^{T} J R=J$ and $R^{T} J R=-J$. A symplectic or anti-symplectic linear operator is necessarily non-singular. We have the following result.

LEMMA 20. The Hamiltonian system (44) is equivariant with respect to a symplectic linear operator $R \in \mathcal{L}\left(\mathbb{R}^{2 N}\right)$ if and only if $H(R \mathbf{x})=$ $H(\mathbf{x})$ for all $\mathbf{x} \in \mathbb{R}^{2 N}$. Similarly, an anti-symplectic linear operator $R \in \mathcal{L}\left(R^{2 N}\right)$ is a reversor of (44) if and only if $H(R \mathbf{x})=H(\mathbf{x})$ for all $\mathbf{x} \in \mathbb{R}^{2 N}$

Proof. We only give the proof for the case of a reversor. The condition for an anti-symplectic $R \in \mathcal{L}\left(\mathbb{R}^{2 N}\right)$ to be a reversor is that $X_{H}(R \mathbf{x})=$ $-R X_{H}(\mathbf{x})$ for all $\mathbf{x} \in \mathbb{R}^{2 N}$, or equivalently, that $\omega\left(X_{H}(R \mathbf{x}), R \tilde{\mathbf{x}}\right)=$ $-\omega\left(R X_{H}(\mathbf{x}), R \tilde{\mathbf{x}}\right)$ for all $\mathbf{x}, \tilde{\mathbf{x}} \in \mathbb{R}^{2 N}$. Since $R$ is anti-symplectic this condition can be rewritten as $\omega\left(X_{H}(R \mathbf{x}), R \tilde{\mathbf{x}}\right)=\omega\left(X_{H}(\mathbf{x}), \tilde{\mathbf{x}}\right)$, or still $D H(R \mathbf{x}) \cdot R \tilde{\mathbf{x}}=D H(\mathbf{x}) \cdot \tilde{\mathbf{x}}$, to be satisfied for all $\mathbf{x}, \tilde{\mathbf{x}} \in \mathbb{R}^{2 N}$. If $H(R \mathbf{x})=H(\mathbf{x})$ then $D H(R \mathbf{x}) \cdot R \tilde{\mathbf{x}}=D H(\mathbf{x}) \cdot \tilde{\mathbf{x}}$ follows by differentiating. Conversely, from $D H(R \mathbf{x}) \cdot R \tilde{\mathbf{x}}=D H(\mathbf{x}) \cdot \tilde{\mathbf{x}}$ we obtain

$$
H(R \mathbf{x})-H(\mathbf{0})=\int_{0}^{1} D H(s R \mathbf{x}) \cdot R \mathbf{x} d s=\int_{0}^{1} D H(s \mathbf{x}) \cdot \mathbf{x} d s ;
$$

since this last integral equals $H(\mathbf{x})-H(\mathbf{0})$ this proves the lemma.

Now suppose that we have two anti-symplectic involutions $R_{0}, R_{1} \in$ $\mathcal{L}\left(\mathbb{R}^{2 N}\right)$ which are reversors of (44), and an element $\mathbf{y}_{0} \in \operatorname{Fix}\left(R_{0}\right)$ which generates a $\left(R_{0}, R_{1}\right)$-symmetric solution $\tilde{\mathbf{x}}_{H}\left(t, \mathbf{y}_{0}\right)$ with basic domain $[0, T]$ and endpoint $\mathbf{z}_{0}=\tilde{\mathbf{x}}_{H}\left(T, \mathbf{y}_{0}\right) \in \operatorname{Fix}\left(R_{1}\right)$; also, let $F: \mathbb{R}^{2 N} \rightarrow \mathbb{R}$ be a first integral of (44). It follows then from (45) that for each $s \in \mathbb{R}$ also $\tilde{\mathbf{x}}_{F}\left(s, \tilde{\mathbf{x}}_{H}\left(t, \mathbf{y}_{0}\right)\right)=\tilde{\mathbf{x}}_{H}\left(t, \tilde{\mathbf{x}}_{F}\left(s, \mathbf{y}_{0}\right)\right)$ is a solution of (44); these solutions 
will again be $\left(R_{0}, R_{1}\right)$-symmetric solutions with basic domain $[0, T]$ if

$$
\tilde{\mathbf{x}}_{F}\left(s, \mathbf{y}_{0}\right) \in \operatorname{Fix}\left(R_{0}\right) \quad \text { and } \quad \tilde{\mathbf{x}}_{F}\left(s, \mathbf{z}_{0}\right) \in \operatorname{Fix}\left(R_{1}\right), \quad \forall s \in \mathbb{R} .
$$

This in turn will be satisfied if both $\operatorname{Fix}\left(R_{0}\right)$ and $\operatorname{Fix}\left(R_{1}\right)$ are invariant under the flow $\tilde{\mathbf{x}}_{F}$ of the Hamiltonian system generated by $F$. The next lemma characterizes those first integrals for which this is the case.

LEMMA 21. Let $R \in \mathcal{L}\left(\mathbb{R}^{2 N}\right)$ be an anti-symplectic involution, and let $F: \mathbb{R}^{2 N} \rightarrow \mathbb{R}$ be of class $\mathscr{C}^{2}$. Then the flow $\tilde{\mathbf{x}}_{F}$ of the Hamiltonian system $\dot{\mathbf{x}}=X_{F}(\mathbf{x})$ leaves $\operatorname{Fix}(R)$ invariant if and only if $F$ is constant on $\operatorname{Fix}(R)$.

Proof. The subspace $\operatorname{Fix}(R)$ is invariant under $\tilde{\mathbf{x}}_{F}$ if and only if for all $\mathbf{y} \in \operatorname{Fix}(R)$ we have $X_{F}(\mathbf{y}) \in \operatorname{Fix}(R)$, which is equivalent to $(I-R) X_{F}(\mathbf{y})=0$ or $R X_{F}(\mathbf{y})=X_{F}(\mathbf{y})$, or still $\omega\left(R X_{F}(\mathbf{y}), \mathbf{x}\right)=$ $\omega\left(X_{F}(\mathbf{y}), \mathbf{x}\right)$ for all $\mathbf{x} \in \mathbb{R}^{2 N}$. Since $R^{2}=I$ and $R$ is anti-symplectic this last condition is equivalent to $\omega\left(X_{F}(\mathbf{y}), R \mathbf{x}\right)=-\omega\left(X_{F}(\mathbf{y}), \mathbf{x}\right)$ for all $\mathbf{x} \in \mathbb{R}^{2 N}$, or $D F(\mathbf{y})(I+R)=0$. This in turn is valid for all $\mathbf{y} \in \operatorname{Fix}(R)$ if and only if $F$ is constant on $\operatorname{Fix}(R)$.

Using Lemma 21 we see that (46) holds for all $F \in \mathscr{F}$. Assuming for simplicity that $n_{0}^{+}=n_{1}^{-}=N$ and choosing $F_{j} \in \mathscr{F}(1 \leq j \leq k)$ as earlier in this section we see that we can obtain a $k$-parameter subfamily of the $(k+1)$-parameter family of $\left(R_{0}, R_{1}\right)$-symmetric solutions to which a normal $\left(R_{0}, R_{1}\right)$-symmetric solution $\tilde{\mathbf{x}}_{H}\left(t, \mathbf{y}_{0}\right)$ belongs by applying the flows $\tilde{\mathbf{x}}_{F_{j}}(1 \leq j \leq k)$ to it. Taking into account the relation between first integrals and symmetries this means that part of the continuation of a doubly symmetric solution can be realized by applying some appropriate symmetry operators. When doing numerical continuation one wants to avoid such "trivial" continuations; this can be achieved by adding to the boundary value problem (41) appropriate "phase conditions" such as

$$
\int_{0}^{1}\left\langle X_{F_{j}}\left(\tilde{\mathbf{x}}_{H}\left(T s, \mathbf{y}_{0}\right)\right),\left(\mathbf{x}(s)-\tilde{\mathbf{x}}_{H}\left(T s, \mathbf{y}_{0}\right)\right)\right\rangle d s=0, \quad(1 \leq j \leq k) .
$$

These conditions should be considered as integral (or "averaged") versions of the more elementary initial conditions

$$
\left\langle X_{F_{j}}\left(\mathbf{y}_{0}\right), \mathbf{x}(0)-\mathbf{y}_{0}\right\rangle=0, \quad(1 \leq j \leq k) .
$$

Adding the conditions (47) or (48) to the boundary value problem (41) reduces the solution set to a one-dimensional curve within the $(k+1)$ dimensional family described above. This has two advantages. First, after discretisation one obtains a regular continuation problem with a 
one-dimensional solution curve which can be followed numerically by (for example) the pseudo-arclength method. And second, the particular form of these additional conditions makes sure that the one-dimensional curve of initial points which one obtains this way is not of the form $s \mapsto \tilde{\mathbf{x}}_{F}\left(s, \mathbf{y}_{0}\right)$ for some $F \in \mathscr{F}$, i.e. one avoids the directions generated by the symmetries of the system. For the practical implementation the phase conditions (47) are preferable over (48) because they seem to provide more reliable results.

The basic step in the continuation scheme goes as follows. Suppose that at some point in the calculation process we have obtained a (normal) $\left(R_{0}, R_{1}\right)$-symmetric solution $\tilde{\mathbf{x}}_{H}\left(t, \mathbf{y}_{0}\right)$ with basic domain $[0, T]$. To determine a nearby such solution we use discretisation and the pseudoarclength method to calculate a number $\tau$ near $T$, a vector $\alpha \in \mathbb{R}^{k}$ near zero, and a mapping $\mathbf{x}(t)(0 \leq t \leq 1)$ near $\tilde{\mathbf{x}}_{H}\left(T t, \mathbf{y}_{0}\right)$ such that $(41)$ (with $X$ replaced by $X_{H}$ ) and (47) are satisfied. From the theory we know that the outcome of this calculation should give us $\alpha=0$, and this is confirmed with high accuracy in all our practical experiments. The new $\left(R_{0}, R_{1}\right)$-symmetric solution (which is the starting point for the next step in the continuation) is then given by $\mathbf{x}(\tau t)=\tilde{\mathbf{x}}_{H}(t, \mathbf{x}(0))$ and has basic domain $[0, \tau]$.

\section{Applications to the N-body problem}

In this section we apply the general results of the foregoing sections and in particular those of subsection 3.4 to some special cases of the $N$-body problem (NBP). In these applications the phase space is $\mathbb{R}^{2 N n}$, with $N$ the number of bodies and with $n=2$ or $n=3$ depending on whether we work in the plane or in 3 -space. The coordinates in this phase space are $\mathbf{x}=\left(\mathbf{q}_{1}, \ldots, \mathbf{q}_{N}, \mathbf{p}_{1}, \ldots, \mathbf{p}_{N}\right)$, with $\mathbf{q}_{j} \in \mathbb{R}^{n}$ and $\mathbf{p}_{j} \in \mathbb{R}^{n}$ respectively the position vector and the momentum vector of the $j$-th body $(1 \leq j \leq N)$. In the space $\mathbb{R}^{n}$ we denote the standard basis by $\left\{\mathbf{e}_{i} \mid 1 \leq i \leq n\right\}$, the standard inner product of $\mathbf{a} \in \mathbb{R}^{n}$ and $\mathbf{b} \in \mathbb{R}^{n}$ by $\mathbf{a} \cdot \mathbf{b}$, and the standard Euclidean norm by $\|\cdot\|$. We will study (some particular cases of) the Hamiltonian system (44) corresponding to the Hamiltonian

$$
H(\mathbf{x})=\sum_{j=1}^{N} \frac{1}{2 m_{j}}\left\|\mathbf{p}_{j}\right\|^{2}-\sum_{1 \leq i<j \leq N} \frac{m_{i} m_{j}}{\left\|\mathbf{q}_{i}-\mathbf{q}_{j}\right\|},
$$

with $m_{j}>0$ the mass of the $j$-th body. 


\subsection{First integrals and Symmetries of the N-BOdy Problem}

Next to the Hamiltonian (49) the system (44) has some further first integrals related by Noether's Theorem to symmetries which leave the Hamiltonian (49) invariant (see Lemma 20). To describe these symmetries we write the elements of the Euclidean group $E(n)$ in the form $(Q, \mathbf{b})$, with $Q \in O(n)$ and $\mathbf{b} \in \mathbb{R}^{n}$. Consider the symplectic action of $E(n)$ on the phase space $\mathbb{R}^{2 N n}$ given by $\Psi_{Q, \mathbf{b}}: \mathbb{R}^{2 N n} \rightarrow \mathbb{R}^{2 N n}$, where

$$
\begin{aligned}
\Psi_{Q, \mathbf{b}}\left(\mathbf{q}_{1}, \ldots, \mathbf{q}_{N}, \mathbf{p}_{1}, \ldots, \mathbf{p}_{N}\right) \\
:=\left(Q \mathbf{q}_{1}+\mathbf{b}, \ldots, Q \mathbf{q}_{N}+\mathbf{b}, Q \mathbf{p}_{1}, \ldots, Q \mathbf{p}_{N}\right) .
\end{aligned}
$$

The Hamiltonian (49) is invariant under this action, and the corresponding first integrals are given by

$$
F_{A, \mathbf{a}}\left(\mathbf{q}_{1}, \ldots, \mathbf{q}_{N}, \mathbf{p}_{1}, \ldots, \mathbf{p}_{N}\right):=\sum_{j=1}^{N} \mathbf{q}_{j} \cdot\left(A \mathbf{p}_{j}\right)+\mathbf{a} \cdot\left(\sum_{j=1}^{N} \mathbf{p}_{j}\right),
$$

with $A \in \mathcal{L}\left(\mathbb{R}^{n}\right)$ skew symmetric and $\mathbf{a} \in \mathbb{R}^{n}$. Including the Hamiltonian his gives us four independent first integrals in the case $n=2$, and seven in the case $n=3$. Moreover, since the total linear momentum $\mathbf{P}:=\sum_{j=1}^{N} \mathbf{p}_{j}$ is a constant of motion we can for each solution find a convenient uniformly moving framework in $\mathbb{R}^{n}$ such that $\mathbf{P}=\mathbf{0}$, which means that the center of mass $\mathbf{Q}:=\sum_{j=1}^{N} m_{j} \mathbf{q}_{j}$ is constant. Throughout the remainder of this section we will mainly restrict to the case $n=2$ and make heavy use of the symmetry operators $\Psi_{1}:=\Psi_{S, \mathbf{0}}$, $\Psi_{2}:=\Psi_{-S, \mathbf{0}}$ and $\Psi_{1,2}:=\Psi_{1} \circ \Psi_{2}=\Psi_{-I, \mathbf{0}}$, where $S \in \mathcal{L}\left(\mathbb{R}^{2}\right)$ is defined by $S \mathbf{e}_{1}:=\mathbf{e}_{1}$ and $S \mathbf{e}_{2}:=-\mathbf{e}_{2}$.

If two or more of the bodies have equal masses then there is a corresponding symmetry which exchanges the positions and the momenta of the bodies with identical masses. For example, if $m_{i}=m_{j}$ for some $i, j$ with $1 \leq i<j \leq N$ then (44) is equivariant with respect to the symplectic operator $C_{i, j} \in \mathcal{L}\left(\mathbb{R}^{2 N n}\right)$ given by

$$
\begin{aligned}
& C_{i, j}\left(\mathbf{q}_{1}, \ldots, \mathbf{q}_{i}, \ldots, \mathbf{q}_{j}, \ldots, \mathbf{q}_{N}, \mathbf{p}_{1}, \ldots, \mathbf{p}_{i}, \ldots, \mathbf{p}_{j}, \ldots, \mathbf{p}_{N}\right) \\
& :=\left(\mathbf{q}_{1}, \ldots, \mathbf{q}_{j}, \ldots, \mathbf{q}_{i}, \ldots, \mathbf{q}_{N}, \mathbf{p}_{1}, \ldots, \mathbf{p}_{j}, \ldots, \mathbf{p}_{i}, \ldots, \mathbf{p}_{N}\right)
\end{aligned}
$$

The Hamiltonian (49) is also invariant under the anti-symplectic operator $R \in \mathcal{L}\left(\mathbb{R}^{2 N n}\right)$ given by

$$
R\left(\mathbf{q}_{1}, \ldots, \mathbf{q}_{N}, \mathbf{p}_{1}, \ldots, \mathbf{p}_{N}\right):=\left(\mathbf{q}_{1}, \ldots, \mathbf{q}_{N},-\mathbf{p}_{1}, \ldots,-\mathbf{p}_{N}\right) .
$$

This implies that $R$ is a time-reversal symmetry (reversor) of (44); moreover, the composition of $R$ with any of the symmetry operators $\Psi_{Q, 0}$ and $C_{i, j}$ forms also a reversor for (44). 
Finally, the $N$-body problem (44) has a rescaling symmetry which plays a role when one considers continuation of solutions. For each $\lambda \in \mathbb{R} \backslash\{0\}$ we define $\Sigma_{\lambda} \in \mathcal{L}\left(\mathbb{R}^{2 N n}\right)$ by

$$
\Sigma_{\lambda}\left(\mathbf{q}_{1}, \ldots, \mathbf{q}_{N}, \mathbf{p}_{1}, \ldots, \mathbf{p}_{N}\right):=\left(\lambda^{-2} \mathbf{q}_{1}, \ldots, \lambda^{-2} \mathbf{q}_{N}, \lambda \mathbf{p}_{1}, \ldots, \lambda \mathbf{p}_{N}\right) .
$$

One can immediately verify that $H\left(\Sigma_{\lambda} \mathbf{x}\right)=\lambda^{2} H(\mathbf{x})$; together with $J \Sigma_{\lambda} J^{T}=\lambda^{-1} \Sigma_{\lambda}^{-1}$ this implies that $\lambda^{3} \Sigma_{\lambda} X_{H}(\mathbf{x})=X_{H}\left(\Sigma_{\lambda} \mathbf{x}\right)$. It follows that for each solution $\hat{\mathbf{x}}(t)$ of (44) and each $\lambda \in \mathbb{R} \backslash\{0\}$ also $\hat{\mathbf{x}}_{\lambda}(t):=$ $\Sigma_{\lambda} \hat{\mathbf{x}}\left(\lambda^{3} t\right)$ forms a solution. Observe that for $\lambda=-1$ this gives us again the reversibility which we discussed before.

\subsection{The FIGURE-EIGHT CHOREOGRAPHY}

As a first example we consider the by now well-known figure-eight solution of the 3 -body problem with equal masses $\left(N=3, m_{1}=m_{2}=\right.$ $\left.m_{3}=1\right)$. This solution is a planar $(n=2)$ periodic solution in which the three bodies perform a choreography by following the same planar curve shaped in the form of an eight. The existence of this curve and the resulting figure-eight solution of the $3 \mathrm{BP}$ with equal masses was obtained in the paper (Chenciner and Montgomery, 2000) by choosing an arbitrary $T>0$ and minimizing the action

$$
\mathcal{A}:=\int_{0}^{T}\left(\frac{1}{2} \sum_{j=1}^{3}\left\|\dot{\mathbf{q}}_{j}(t)\right\|^{2}+\sum_{1 \leq i<j \leq 3} \frac{1}{\left\|\mathbf{q}_{i}(t)-\mathbf{q}_{j}(t)\right\|}\right) d t,
$$

over the space of differentiable paths $\mathbf{q}:[0, T] \rightarrow \mathcal{X}$ in the configuration space $\mathcal{X}:=\left\{\mathbf{q}=\left(\mathbf{q}_{1}, \mathbf{q}_{2}, \mathbf{q}_{3}\right) \in\left(\mathbb{R}^{2}\right)^{3} \mid \mathbf{q}_{1}+\mathbf{q}_{2}+\mathbf{q}_{3}=\mathbf{0}\right\}$ which connect the following start and end configurations of the three bodies:

- Start configuration: the three bodies are on a straight line, with the second body in the middle (a so-called Euler configuration).

- End configuration: the three bodies form an isosceles triangle with the first body (which was in an extremal position at the start) on the symmetry axis.

It is shown in (Chenciner and Montgomery, 2000) that such minimizing path exists, that it is collisionless, and that by symmetry it can be extended to a $12 T$-periodic solution $\mathbf{x}_{0}(t)$ of the $3 \mathrm{BP}$ with zero angular momentum and with the choreographic features described above. Figure 2 shows the figure-eight curve followed by the three bodies and indicates the start and end configurations as realized in the minimizing path. Observe that the figure-eight orbits are determined only up to 


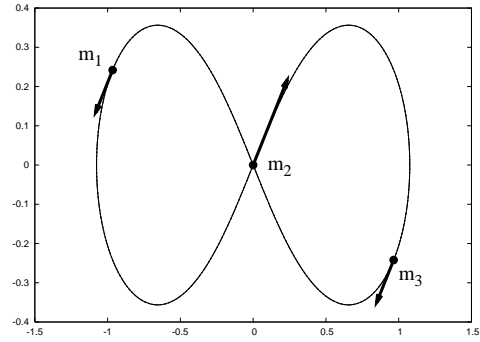

Start configuration

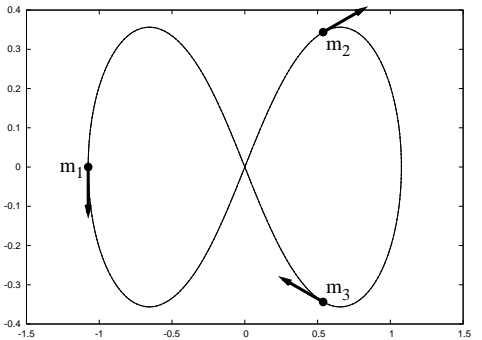

End configuration

Figure 2. The figure-eight choreography with the start and end configurations. The bodies move as indicated by the arrows; the time between the start and the end configuration is one twelfth of the total period.

the symmetries of the system, and therefore Figure 2 shows only one particular realization of these orbits. Moreover, using the scaling symmetry on a figure-eight orbit leads again to a figure-eight orbit, however with a different value of $T$.

When one wants to continue the figure-eight solution $\mathbf{x}_{0}(t)$ several approaches are possible which we discuss now briefly.

(1) Analyzing the symmetries of the figure-eight solution one finds that it actually is a doubly symmetric solution with basic domain $[0, T]$, as

follows. Because of the equal masses we have next to the symmetries (50) and the reversibility also the exchange symmetries $C_{1,2}, C_{1,3}$ and $C_{2,3}$. Using these symmetries one observes that on the interval $[0, T]$ the particular figure-eight solution $\mathbf{x}_{0}(t)$ as depicted in Figure 2 satisfies the following conditions:

- Start configuration: $\mathbf{x}_{0}(0) \in \operatorname{Fix}\left(R \circ C_{1,3} \circ \Psi_{1,2}\right)$, i.e. at time $t=0$ we have $\mathbf{q}_{2}=\mathbf{0}, \mathbf{q}_{1}=-\mathbf{q}_{3}$ and $\mathbf{p}_{1}=\mathbf{p}_{3}$.

- End configuration: $\mathbf{x}_{0}(T) \in \operatorname{Fix}\left(R \circ C_{2,3} \circ \Psi_{1}\right)$, i.e. at time $t=T$ we have $\mathbf{q}_{1}=S \mathbf{q}_{1}, \mathbf{q}_{2}=S \mathbf{q}_{3}, \mathbf{p}_{1}=-S \mathbf{p}_{1}$ and $\mathbf{p}_{2}=-S \mathbf{p}_{3}$.

We conclude that $\mathbf{x}_{0}(t)$ is a $\left(R_{0}, R_{1}\right)$-symmetric solution, with

$$
R_{0}:=R \circ C_{1,3}{ }^{\circ} \Psi_{1,2} \quad \text { and } \quad R_{1}:=R \circ C_{2,3}{ }^{\circ} \Psi_{1} .
$$

Explicitly $R_{0}$ and $R_{1}$ are given by

$$
\left\{\begin{array}{l}
R_{0}\left(\mathbf{q}_{1}, \mathbf{q}_{2}, \mathbf{q}_{3}, \mathbf{p}_{1}, \mathbf{p}_{2}, \mathbf{p}_{3}\right)=\left(-\mathbf{q}_{3},-\mathbf{q}_{2},-\mathbf{q}_{1}, \mathbf{p}_{3}, \mathbf{p}_{2}, \mathbf{p}_{1}\right), \\
R_{1}\left(\mathbf{q}_{1}, \mathbf{q}_{2}, \mathbf{q}_{3}, \mathbf{p}_{1}, \mathbf{p}_{2}, \mathbf{p}_{3}\right)=\left(S \mathbf{q}_{1}, S \mathbf{q}_{3}, S \mathbf{q}_{2},-S \mathbf{p}_{1},-S \mathbf{p}_{3},-S \mathbf{p}_{2}\right) .
\end{array}\right.
$$

It follows that

$$
\left(R_{1} R_{0}\right)\left(\mathbf{q}_{1}, \mathbf{q}_{2}, \mathbf{q}_{3}, \mathbf{p}_{1}, \mathbf{p}_{2}, \mathbf{p}_{3}\right)=\left(-S \mathbf{q}_{3},-S \mathbf{q}_{1},-S \mathbf{q}_{2},-S \mathbf{p}_{3},-S \mathbf{p}_{1},-S \mathbf{p}_{2}\right)
$$


and $\left(R_{1} R_{0}\right)^{6}=I$; hence each $\left(R_{0}, R_{1}\right)$-symmetric solution with basic domain $[0, T]$ is automatically $12 T$-periodic. As we have seen this is in particular the case for the figure-eight solution. Also $\operatorname{dim} \operatorname{Fix}\left(R_{0}\right)=$ $\operatorname{dim} \operatorname{Fix}\left(-R_{0}\right)=\operatorname{dim} \operatorname{Fix}\left(R_{1}\right)=\operatorname{dim} \operatorname{Fix}\left(-R_{1}\right)=6$, i.e. $n_{0}^{+}=n_{0}^{-}=$ $n_{1}^{+}=n_{1}^{-}=6$.

One can easily verify that neither the Hamiltonian $H$ nor any of the first integrals $F_{A, \mathbf{a}}$ given by (51) are constant on $\operatorname{Fix}\left(R_{0}\right) \cup \operatorname{Fix}\left(R_{1}\right)$; so $k=0$. Assuming that $\mathbf{x}_{0}(t)$ is normal (we checked numerically that this is indeed the case) it follows from the theory of Section 3 that this solution belongs to a one-parameter family of $\left(R_{0}, R_{1}\right)$-symmetric orbits. From the preceding discussion we see that this family can be nothing else but the family of rescaled versions of $\mathbf{x}_{0}(t)$; therefore, continuing the figure-eight orbit as a doubly symmetric solution does not reveal much news.

(2) The figure-eight solution $\mathbf{x}_{0}(t)$ is also a $\left(R_{0}, R_{0}\right)$-symmetric solution with basic domain $[0,6 T]$, that is, a $R_{0}$-symmetric periodic solution. When we want to continue $\mathbf{x}_{0}(t)$ as a $R_{0}$-symmetric periodic orbit we have to set $k=1$ since there is one independent first integral which is constant on $\operatorname{Fix}\left(R_{0}\right)$, namely the total angular momentum $F_{0}(\mathbf{x}):=\sum_{j=1}^{3} \mathbf{q}_{j} \cdot\left(A \mathbf{p}_{j}\right)$, with $A \mathbf{e}_{1}:=-\mathbf{e}_{2}$ and $A \mathbf{e}_{2}:=\mathbf{e}_{1}$. Therefore $\mathbf{x}_{0}(t)$ belongs to a 2 -parameter family of $R_{0}$-symmetric periodic orbits; one obtains this family by scaling and by rotation around the origin. In the PhD-thesis (Muñoz Almaraz, 2003) and in the paper (Muñoz Almaraz et al., 2004) one can find the numerical results of such continuation when also the mass $m_{2}$ of the second body (which takes the central position in the Euler configurations at $t=0$ and $t=6 T$ ) is allowed to vary; observe that $R_{0}$ remains a reversor if $m_{2} \neq 1$ but $m_{1}=m_{3}=1$. With the additional parameter the manifold of $R_{0^{-}}$ symmetric period orbits becomes 3-dimensional; in order to reduce this to a one-dimensional curve suitable for numerical calculations one has to fix $T$ in the boundary value problem (see (38))

$$
\left\{\begin{array}{l}
\dot{\mathbf{x}}=X_{H}(\mathbf{x})+\alpha \nabla F_{0}(\mathbf{x}), \\
\mathbf{x}(0) \in \operatorname{Fix}\left(R_{0}\right), \quad \mathbf{x}(6 T) \in \operatorname{Fix}\left(R_{0}\right),
\end{array}\right.
$$

and add to (57) a phase condition similar to (47) which here takes the form

$$
\int_{0}^{6 T} X_{F_{0}}\left(\mathbf{x}_{0}(t)\right) \cdot\left(\mathbf{x}(t)-\mathbf{x}_{0}(t)\right) d t=0 .
$$

(Fixing $T$ blocks the scalings, while (58) prevents rotations). As explained in subsection 3.3 all solutions of (57) will have $\alpha=0$. We refer to (Muñoz Almaraz, 2003) and (Muñoz Almaraz et al., 2004) for more details and for some pictures of the $R_{0}$-symmetric periodic orbits 
resulting from such continuation. Observe that in (57) we did not use a time rescale such as in (41) because with $T$ fixed this does not give any advantage.

(3) In the same way one can consider the figure-eight solution $\mathbf{x}_{0}(t)$ as a $\left(R_{1}, R_{1}\right)$-symmetric solution with basic domain $[T, 7 T]$. Then again one has $k=1$, corresponding to the first integral $P_{1}(\mathbf{x}):=\mathbf{e}_{1} \cdot\left(\mathbf{p}_{1}+\mathbf{p}_{2}+\mathbf{p}_{3}\right)$ (the first component of the total linear momentum); the resulting 2dimensional family of $R_{1}$-symmetric periodic solutions is obtained from $\mathbf{x}_{0}(t)$ by rescaling and by translations in the $\mathbf{e}_{1}$-direction. In this case one is allowed to change the mass $m_{1}$ of the first body without destroying $R_{1}$ as a reversor; we refer again to (Muñoz Almaraz, 2003) and (Muñoz Almaraz et al., 2004) for numerical results from a continuation along these lines.

(4) Finally one can ignore all particular symmetries and consider $\mathbf{x}_{0}(t)$ as a plain $12 T$-periodic orbit. Then the continuation theory of (Muñoz Almaraz et al., 2003) is applicable and will involve all 4 independent first integrals (or all 7 integrals if we consider the continuation problem in 3-space). We refer to (Doedel et al., 2003) for some results originating from such approach.

We conclude this subsection with two remarks. The first is that when we keep all masses fixed and equal the one-dimensional family of $\left(R_{0}, R_{1}\right)$-symmetric solutions resulting from the approach (1) can also be obtained by intersecting the two-parameter family of $R_{0}$-symmetric periodic orbits resulting from the approach (2) with the two-parameter family of $R_{1}$-symmetric periodic orbits resulting from the approach (3). A second remark is that in (Kapela and Zgliczyński, 2003) it is shown that for equal masses the figure-eight solution is locally unique up to obvious symmetries; assuming normality our results agree with that conclusion. This agreement seems to be a confirmation of that normality (which we could only check numerically).

\subsection{Gerver's supereight Choreography}

The so-called "supereight solution" which we study in this subsection is one of the simplest choreographies after the Lagrange solution and the figure-eight solution; it is a planar choreography for four bodies with equal mass $\left(N=4, m_{1}=m_{2}=m_{3}=m_{4}=1\right)$ which follow a curve in the form of an eight which has grown an additional bubble at its center - see Figure 3. This choreography was discovered numerically by Gerver, and its existence was proved using a computer assisted approach in (Kapela and Zgliczyński, 2003). We denote the particular supereight solution as depicted in Figure 3 by $\mathbf{x}_{0}(t)=\left(\mathbf{q}_{0, j}(t), \mathbf{p}_{0, j}(t)\right)$, and the time between the start and the end configuration by $T>0$. 


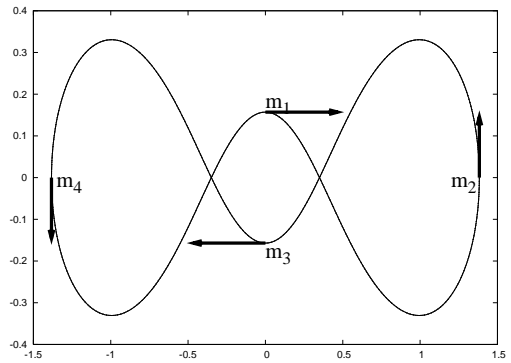

Start configuration

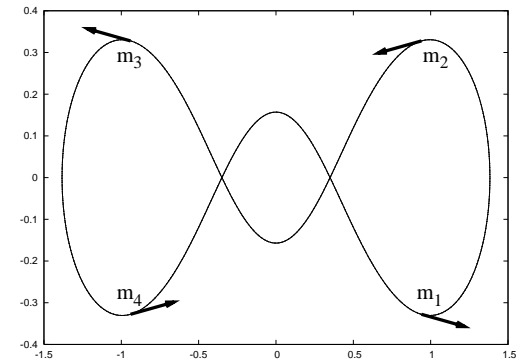

End configuration

Figure 3. Gerver's supereight choreography. The time between the start and the end configuration is one eighth of the full period.

The start configuration as depicted in Figure 3 belongs to $\operatorname{Fix}\left(R_{0}\right)$, with the reversor $R_{0}$ given by $R_{0}=R \circ C_{1,3}{ }^{\circ} \Psi_{1}$. We have

$$
R_{0} \mathbf{x}=\left(S \mathbf{q}_{3}, S \mathbf{q}_{2}, S \mathbf{q}_{1}, S \mathbf{q}_{4},-S \mathbf{p}_{3},-S \mathbf{p}_{2},-S \mathbf{p}_{1},-S \mathbf{p}_{4}\right)
$$

and $\mathbf{x}=\left(\mathbf{q}_{1}, \mathbf{q}_{2}, \mathbf{q}_{3}, \mathbf{q}_{4}, \mathbf{p}_{1}, \mathbf{p}_{2}, \mathbf{p}_{3}, \mathbf{p}_{4}\right)$ belongs to $\operatorname{Fix}\left(R_{0}\right)$ if and only if $\mathbf{q}_{1}=S \mathbf{q}_{3}, S \mathbf{q}_{2}=\mathbf{q}_{2}, S \mathbf{q}_{4}=\mathbf{q}_{4}, \mathbf{p}_{1}=-S \mathbf{p}_{3}, \mathbf{p}_{2}=-S \mathbf{p}_{2}, \mathbf{p}_{4}=-S \mathbf{p}_{4}$.

The end configuration belongs to $\operatorname{Fix}\left(R_{1}\right)$, with $R_{1}=R \circ C_{1,2}{ }^{\circ} C_{3,4} \circ \Psi_{1}$; explicitly the reversor $R_{1}$ is given by

$$
R_{1} \mathbf{x}=\left(S \mathbf{q}_{2}, S \mathbf{q}_{1}, S \mathbf{q}_{4}, S \mathbf{q}_{3},-S \mathbf{p}_{2},-S \mathbf{p}_{1},-S \mathbf{p}_{4},-S \mathbf{p}_{3}\right),
$$

and $\operatorname{Fix}\left(R_{1}\right)$ is determined by the conditions

$$
\mathbf{q}_{1}=S \mathbf{q}_{2}, \mathbf{q}_{3}=S \mathbf{q}_{4}, \mathbf{p}_{1}=-S \mathbf{p}_{2}, \mathbf{p}_{3}=-S \mathbf{p}_{4} .
$$

It follows that

$$
R_{1} R_{0} \mathbf{x}=\left(\mathbf{q}_{2}, \mathbf{q}_{3}, \mathbf{q}_{4}, \mathbf{q}_{1}, \mathbf{p}_{2}, \mathbf{p}_{3}, \mathbf{p}_{4}, \mathbf{p}_{1}\right) \quad \Rightarrow \quad\left(R_{1} R_{0}\right)^{4}=I,
$$

and therefore each $\left(R_{0}, R_{1}\right)$-symmetric solution with basic domain $[0, T]$ is automatically $8 T$-periodic. This holds in particular for the supereight choreography. Also $n_{0}^{+}=n_{0}^{-}=n_{1}^{+}=n_{1}^{-}=8$. Up to multiplication with a scalar the only first integral which is constant on $\operatorname{Fix}\left(R_{0}\right) \cup \operatorname{Fix}\left(R_{1}\right)$ is the $\mathbf{e}_{1}$-component of the total linear momentum $P_{1}(\mathbf{x}):=\mathbf{e}_{1} \cdot\left(\mathbf{p}_{1}+\right.$ $\left.\mathbf{p}_{2}+\mathbf{p}_{3}+\mathbf{p}_{4}\right)$.

We have checked numerically that the supereight solution is normal as a $\left(R_{0}, R_{1}\right)$-symmetric solution. Therefore it belongs to a twoparameter family $(k=1)$ of such doubly symmetric solutions; this 
family can be obtained from $\mathbf{x}_{0}(t)$ by the rescalings $\Sigma_{\lambda}$ and by translations in the $\mathbf{e}_{1}$-direction. In order to obtain some nontrivial continuation one needs an additional parameter in the Hamiltonian. We cannot change one or several of the masses since the four masses must be equal for both $R_{0}$ and $R_{1}$ to remain reversors. Instead we will allow the potential to change by considering the following modified Hamiltonian:

$$
H_{\gamma}(\mathbf{x})=\frac{1}{2} \sum_{j=1}^{4}\left\|\mathbf{p}_{j}\right\|^{2}-\sum_{1 \leq i<j \leq 4}^{4} \frac{1}{\left\|\mathbf{q}_{j}-\mathbf{q}_{i}\right\|^{\gamma}}, \quad \text { with } \gamma>0 .
$$

For $\gamma=1$ one gets the classical Newtonian potential, for $\gamma<1$ one speaks about weak forces and for $\gamma>1$ about strong forces. In recent studies on choreographic motions it has become more or less standard to consider this type of potential, see e.g. (Ferrario and Terracini, 2004) or (Moore, 1993); in this last paper it is for example shown that no collisions can appear for $\gamma \geq 2$.

Starting from the supereight solution $\mathbf{x}_{0}(t)$ at $\gamma=1$ we obtain now a three-dimensional continuation manifold. In order to prevent rescalings and translations in the $\mathbf{e}_{1}$-direction one fixes $T$ in the boundary value problem

$$
\left\{\begin{array}{l}
\dot{\mathbf{x}}=X_{H_{\gamma}}(\mathbf{x})+\alpha \nabla P_{1}(\mathbf{x}), \\
\mathbf{x}(0) \in \operatorname{Fix}\left(R_{0}\right), \quad \mathbf{x}(T) \in \operatorname{Fix}\left(R_{1}\right),
\end{array}\right.
$$

(compare with (38) and (41)), and one adds to (60) the phase condition

$$
\int_{0}^{T} \mathbf{e}_{1} \cdot\left(\sum_{j=1}^{4}\left(\mathbf{q}_{j}(t)-\mathbf{q}_{0, j}(t)\right)\right) d t=0 .
$$

The result is then a one-parameter family of $\left(R_{0}, R_{1}\right)$-symmetric solutions parametrized by $\gamma$; in Figure 4 we have plotted some of the orbits which belong to that family. All the solutions along the calculated branch (ranging from approximately $\gamma=0.5$ to $\gamma=2.0$ ) are choreographies of supereight type, and we did not find any bifurcation of doubly-symmetric solutions along the branch.

The supereight solution $\mathbf{x}_{0}(t)$ can also be considered as a $\left(R_{0}, R_{0}\right)$ symmetric solution with basic domain $[0,4 T]$; as such the solution is normal (checked numerically), and it belongs to a two-parameter family of $\left(R_{0}, R_{0}\right)$-symmetric solutions obtained from $\mathbf{x}_{0}(t)$ by rescaling and translation in the $\mathbf{e}_{1}$-direction. In this case we are allowed to change some of the masses without destroying $R_{0}$ as a reversor. More in particular, $R_{0}$ remains a reversor as long as $m_{1}=m_{3}$; here we will consider the case where $m_{1}=m_{3}=m$ and $m_{2}=m_{4}=1$. The corresponding 

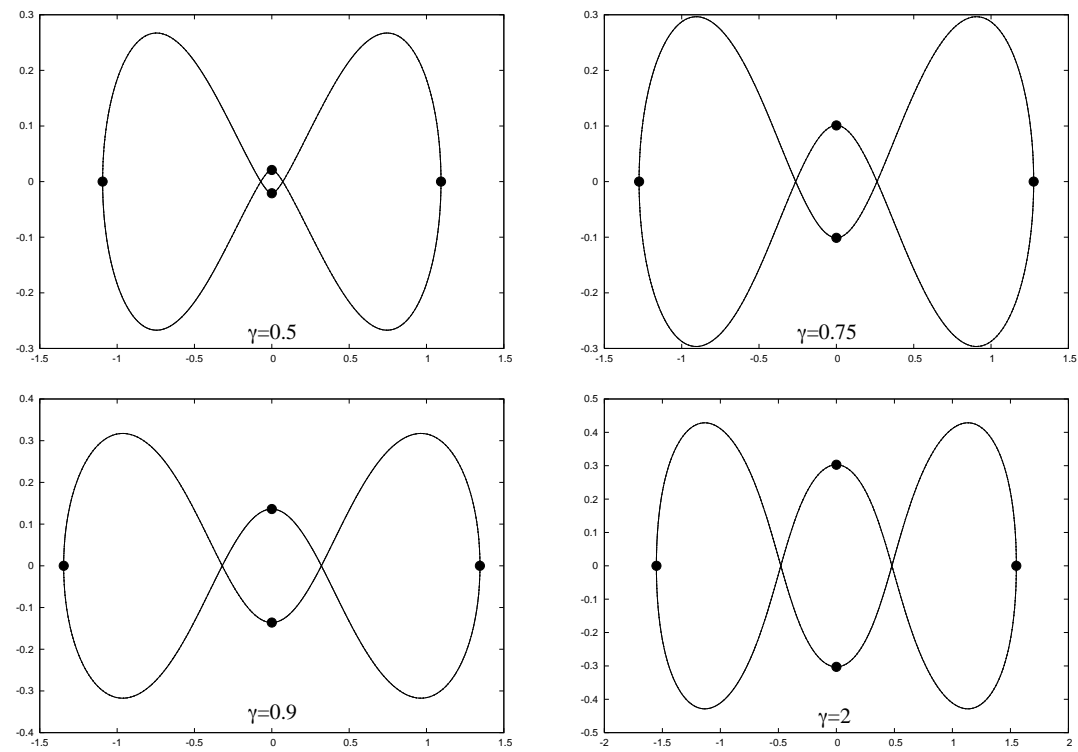

\begin{tabular}{|l|l|l|l|l|}
\hline$\gamma$ & $\mathbf{q}_{1}=-\mathbf{q}_{3}$ & $\mathbf{q}_{2}=-\mathbf{q}_{4}$ & $\mathbf{p}_{1}=-\mathbf{p}_{3}$ & $\mathbf{p}_{2}=-\mathbf{p}_{4}$ \\
\hline 0.49841279 & $(0,0.02084342)$ & $(1.09374645,0)$ & $(2.14172893,0)$ & $(0,0.46372448)$ \\
\hline 0.75 & $(0,0.10100571)$ & $(1.27537449,0)$ & $(1.83317935,0)$ & $(0,0.52206750)$ \\
\hline 0.9 & $(0,0.13606289)$ & $(1.34564508,0)$ & $(1.85485689,0)$ & $(0,0.56052240)$ \\
\hline 2.0 & $(0,0.30290884)$ & $(1.55127326,0)$ & $(1.96007579,0)$ & $(0,0.76669781)$ \\
\hline
\end{tabular}

Figure 4. Some $\left(R_{0}, R_{1}\right)$-symmetric orbits for the modified Hamiltonian $H_{\gamma}$. The table contains the data for the initial conditions. Observe that some of the zeros in these initial data are not due to the condition that the initial point should belong to $\operatorname{Fix}\left(R_{0}\right)$; indeed, as explained in the text there is an additional symmetry which, according to the calculations, is preserved along the full branch.

Hamiltonian depends on the parameter $m$ and takes the explicit form

$$
\begin{gathered}
H_{m}(\mathbf{x})=\frac{1}{2 m}\left(\left\|\mathbf{p}_{1}\right\|^{2}+\left\|\mathbf{p}_{3}\right\|^{2}\right)+\frac{1}{2}\left(\left\|\mathbf{p}_{2}\right\|^{2}+\left\|\mathbf{p}_{4}\right\|^{2}\right)-\frac{m^{2}}{\left\|\mathbf{q}_{3}-\mathbf{q}_{1}\right\|} \\
-\frac{m}{\left\|\mathbf{q}_{2}-\mathbf{q}_{1}\right\|}-\frac{m}{\left\|\mathbf{q}_{4}-\mathbf{q}_{1}\right\|}-\frac{m}{\left\|\mathbf{q}_{3}-\mathbf{q}_{2}\right\|}-\frac{m}{\left\|\mathbf{q}_{4}-\mathbf{q}_{3}\right\|}-\frac{1}{\left\|\mathbf{q}_{4}-\mathbf{q}_{2}\right\|}
\end{gathered}
$$

In order to calculate a one-dimensional continuation branch parametrized by $m$ we use this time the boundary value problem and phase condition

$$
\left\{\begin{array}{l}
\dot{\mathbf{x}}=X_{H_{m}}(\mathbf{x})+\alpha \nabla P_{1}(\mathbf{x}) \\
\mathbf{x}(0) \in \operatorname{Fix}\left(R_{0}\right), \mathbf{x}(4 T) \in \operatorname{Fix}\left(R_{0}\right) \\
\int_{0}^{4 T} \mathbf{e}_{1} \cdot\left(\sum_{j=1}^{4}\left(\mathbf{q}_{j}(t)-\mathbf{q}_{0, j}(t)\right)\right) d t=0
\end{array}\right.
$$



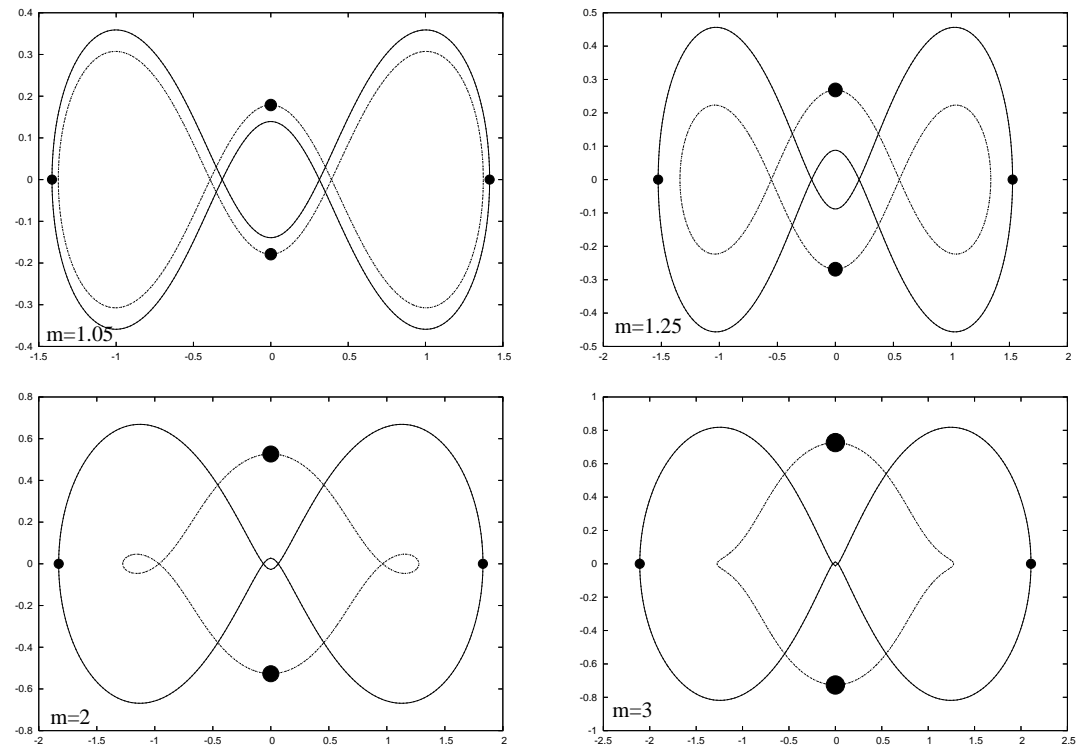

\begin{tabular}{|l|l|l|l|l|}
\hline$m$ & $\mathbf{q}_{1}=-\mathbf{q}_{3}$ & $\mathbf{q}_{2}=-\mathbf{q}_{4}$ & $\mathbf{p}_{1}=-\mathbf{p}_{3}$ & $\mathbf{p}_{2}=-\mathbf{p}_{4}$ \\
\hline 1.05 & $(0,0.17895335)$ & $(1.41447225,0)$ & $(1.77885725,0)$ & $(0,0.61358962)$ \\
\hline 1.25 & $(0,0.26865463)$ & $(1.52729190,0)$ & $(1.51294344,0)$ & $(0,0.70034331)$ \\
\hline 2.0 & $(0,0.52669524)$ & $(1.82851084,0)$ & $(1.19062223,0)$ & $(0,0.84295753)$ \\
\hline 3.0 & $(0,0.72626717)$ & $(2.10748027,0)$ & $(1.14363919,0)$ & $(0,0.93320427)$ \\
\hline
\end{tabular}

Figure 5. Some $R_{0}$-symmetric periodic orbits for the modified Hamiltonian $H_{m}$. The table contains the initial data; the observation in Figure 4 about additional symmetries also applies here.

in which we keep $T$ fixed and use $m$ as the continuation parameter. Figure 5 shows some of the orbits along this branch. Observe that for $m \neq 1$ the orbits are no longer choreographies, but only "partial choreographies" in which the first and the third body follow one curve while the second and the fourth body follow a different curve.

Along the foregoing branch of $R_{0}$-symmetric periodic orbits we found only one point, namely at $m=0.712412 \ldots$, where the solution is not normal and where there is a bifurcation of an other branch of $R_{0^{-}}$ symmetric periodic orbits. The orbits along this bifurcating branch are shown in Figure 6 and are no longer choreographies or partial choreographies.

Next we observe that the supereight solution $\mathbf{x}_{0}(t)$ has more symmetries than the ones we have exploited so far. Indeed, we can also consider it as a $\left(\tilde{R}_{0}, \tilde{R}_{0}\right)$-symmetric solution with basic domain $[0,4 T]$, where $\tilde{R}_{0}:=R \circ C_{2,4} \circ \Psi_{2}$ is a reversor for the system corresponding to the original $N$-body Hamiltonian (49) with $N=4$ and $m_{1}=m_{2}=$ 

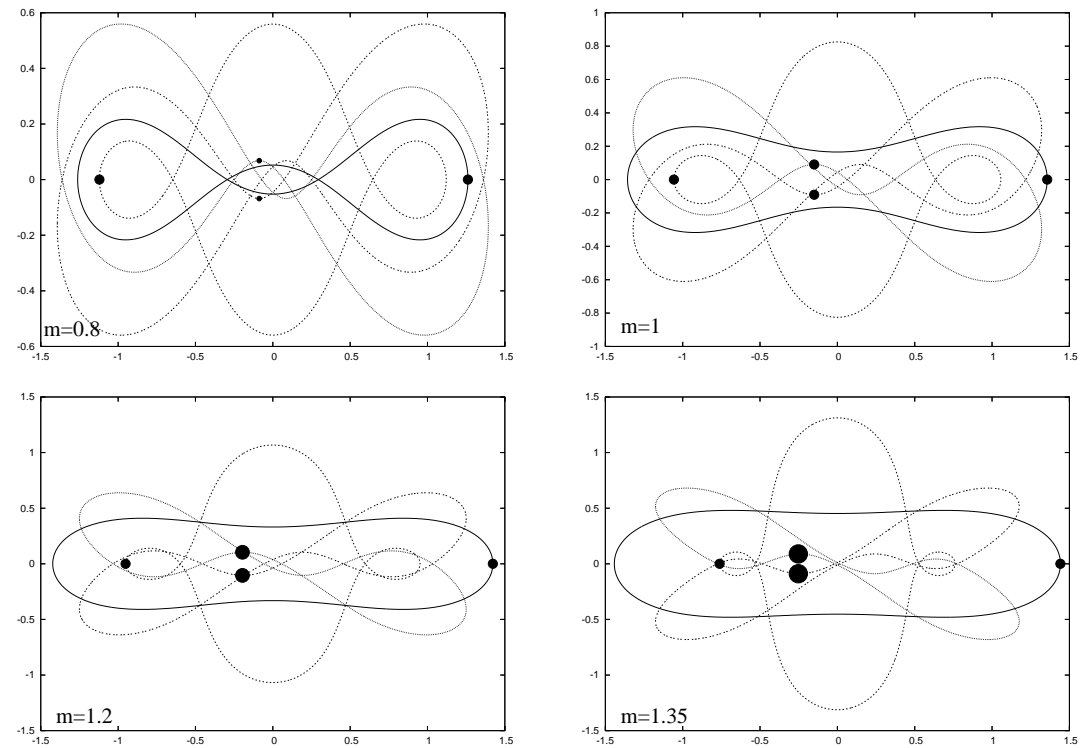

\begin{tabular}{|l|l|l|}
\hline$m$ & Positions & Momenta \\
\hline 0.8 & $\mathbf{q}_{1}=(-0.08770812,0.06825114)$ & $\mathbf{p}_{1}=\left(2.62800497,4.12978 \cdot 10^{-04}\right)$ \\
& $\mathbf{q}_{2}=(1.26150883,0)$ & $\mathbf{p}_{2}=(0,0.41027206)$ \\
& $\mathbf{q}_{4}=(-1.12117587,0)$ & $\mathbf{p}_{4}=(0,-0.41093283)$ \\
\hline 1.0 & $\mathbf{q}_{1}=(-0.14986384,0.09067694)$ & $\mathbf{p}_{1}=(2.56447225,0.01303443)$ \\
& $\mathbf{q}_{2}=(1.35655728,0)$ & $\mathbf{p}_{2}=(0,0.52120812)$ \\
& $\mathbf{q}_{4}=(-1.05682961,0)$ & $\mathbf{p}_{4}=(0,-0.54727698)$ \\
\hline 1.2 & $\mathbf{q}_{1}=(-0.19603803,0.10393649)$ & $\mathbf{p}_{1}=(2.65817327,0.04426818)$ \\
& $\mathbf{q}_{2}=(1.42262247,0)$ & $\mathbf{p}_{2}=(0,0.61904620)$ \\
& $\mathbf{q}_{4}=(-0.95213120,0)$ & $\mathbf{p}_{4}=(0,-0.72528982)$ \\
\hline 1.35 & $\mathbf{q}_{1}=(-0.25207756,0.08866794)$ & $\mathbf{p}_{1}=(3.14956529,0.12518335)$ \\
& $\mathbf{q}_{2}=(1.44197577,0)$ & $\mathbf{p}_{2}=(0,0.68047109)$ \\
& $\mathbf{q}_{4}=(-0.76136634,0)$ & $\mathbf{p}_{4}=(0,-1.01846612)$ \\
\hline
\end{tabular}

Figure 6 . Some of the $R_{0}$-symmetric periodic orbits for the modified Hamiltonian $H_{m}$ along the branch bifurcating at $m=0.712412 \ldots$, and the corresponding initial data; in the table we have not included $\mathbf{q}_{3}=S \mathbf{q}_{1}$ and $\mathbf{p}_{3}=-S \mathbf{p}_{1}$.

$m_{3}=m_{4}=1$, and remains so for the Hamiltonians $H_{m}$ given by (62). Up to multiplication by a scalar the only first integral which is constant on $\operatorname{Fix}\left(\tilde{R}_{0}\right)$ is $P_{2}(\mathbf{x}):=\mathbf{e}_{2} \cdot\left(\mathbf{p}_{1}+\mathbf{p}_{2}+\mathbf{p}_{3}+\mathbf{p}_{4}\right)$ (the second component of the total linear momentum). When we keep $m=1$ we see that $\mathbf{x}_{0}(t)$ belongs to a two-parameter family of $\tilde{R}_{0}$-symmetric periodic solutions, obtained from $\mathbf{x}_{0}(t)$ by rescaling and translations in the $\mathbf{e}_{2}$-direction. By keeping $T$ fixed and allowing $m$ to vary in the boundary value 
problem

$$
\left\{\begin{array}{l}
\dot{\mathbf{x}}=X_{H_{m}}(\mathbf{x})+\alpha \nabla P_{2}(\mathbf{x}), \\
\mathbf{x}(0) \in \operatorname{Fix}\left(\tilde{R}_{0}\right), \mathbf{x}(4 T) \in \operatorname{Fix}\left(\tilde{R}_{0}\right), \\
\int_{0}^{4 T} \mathbf{e}_{2} \cdot\left(\sum_{j=1}^{4}\left(\mathbf{q}_{j}(t)-\mathbf{q}_{0, j}(t)\right)\right) d t=0
\end{array}\right.
$$

we obtain a one-dimensional branch of $\tilde{R}_{0}$-symmetric periodic orbits which coincides with the branch of $R_{0}$-symmetric periodic orbits found by using (63) and which is illustrated in Figure 5. This implies that the solutions $\mathbf{x}_{m}(t)$ along this branch are such that $\mathbf{x}_{m}(0) \in \operatorname{Fix}\left(R_{0}\right) \cap$ $\operatorname{Fix}\left(\tilde{R}_{0}\right) \subset \operatorname{Fix}\left(\tilde{R}_{0} R_{0}\right)$, and since the vectorfield $X_{H_{m}}$ commutes with $\tilde{R}_{0} R_{0}$ it follows that $\mathbf{x}_{m}(t)=\tilde{R}_{0} R_{0} \mathbf{x}_{m}(t)$, or more explicitly $\mathbf{x}_{m}(t)=$ $-C_{1,3} C_{2,4} \mathbf{x}_{m}(t)$ for all $t \in \mathbb{R}$. This is precisely the symmetry which is illustrated in Figure 5 and the accompanying table. The additional $\tilde{R}_{0^{-}}$ symmetry is not present in the branch bifurcating at $m=0.712412 \ldots$ and shown in Figure 6 (i.e. the $\tilde{R}_{0}$-symmetry is broken at the bifurcation), but it is present along the solution branch obtained from (60)-(61) and depicted in Figure 4.

Continuing the supereight solution $\mathbf{x}_{0}(t)$ using the scheme (64) we find different bifurcation points than the one obtained by using the scheme (63). The bifurcation points are now at $m=0.253431 \ldots, m=$ $0.685285 \ldots, m=1.403682 \ldots, m=1.459246 \ldots$ and $m=3.945835 \ldots$, and at each of these points a branch of $\tilde{R}_{0}$-symmetric periodic orbits bifurcates from the branch $\left\{\mathbf{x}_{m} \mid m>0\right\}$; actually, the branches bifurcating at $m=0.253431 \ldots$ and at $m=0.685285 \ldots$ are connected to each other, and the same is true for the branches bifurcating at $m=1.459246 \ldots$ and at $m=3.945835 \ldots$. The periodic orbits along these bifurcating branches do not have the $R_{0}$-symmetry. In Figure 7 some of the orbits along the branch connecting $m=1.459246 \ldots$ to $m=3.945835 \ldots$ are shown; the solutions along this branch are partial choreographies, with the first and third bodies following one curve, while the second and fourth bodies follow a different curve.

Finally we can also consider the supereight solution $\mathbf{x}_{0}(t)$ as a periodic orbit of a Hamiltonian systems with first integrals and use the continuation scheme of (Muñoz Almaraz et al., 2003). Using this approach (for the Hamiltonian $H_{m}$ ) we found again the same branch as obtained using the schemes (63) or (64), and no further bifurcation points were detected. We have obtained several other families of symmetric periodic orbits starting from Gerver's supereight solution, for example by changing only one of the masses; we will report on these results in a later paper. 

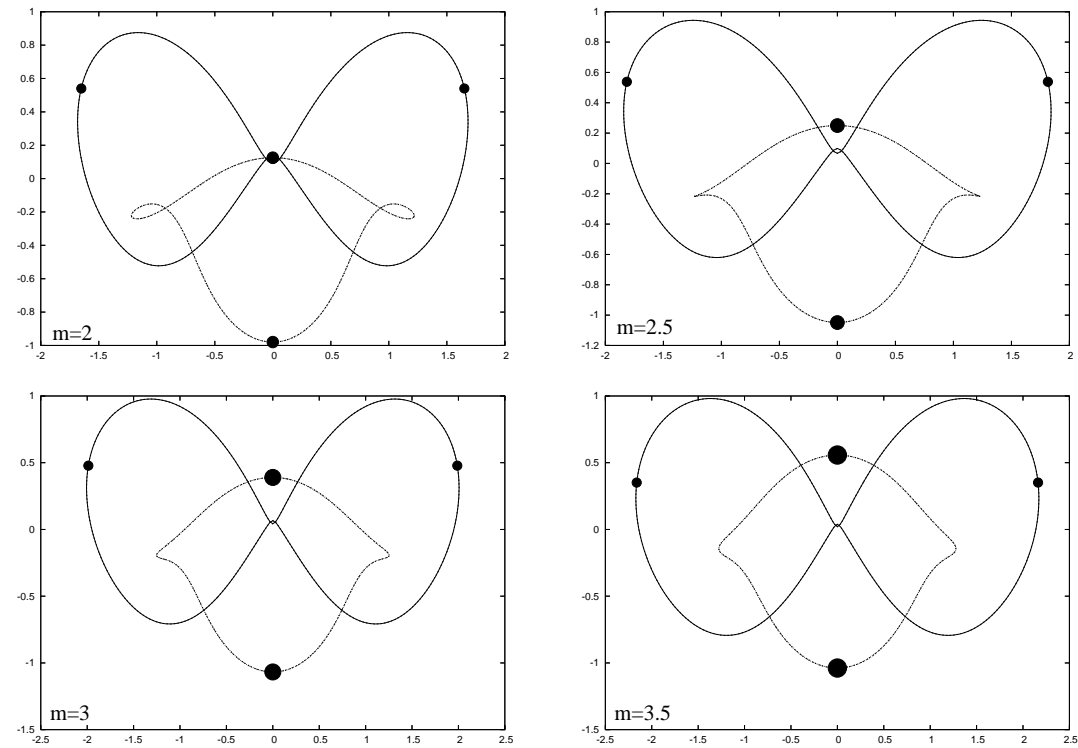

\begin{tabular}{|l|l|l|}
\hline$m$ & Positions & Momenta \\
\hline 2.0 & $\mathbf{q}_{1}=(0,0.12560906)$ & $\mathbf{p}_{1}=(1.28285805,0)$ \\
& $\mathbf{q}_{2}=(1.65151910,0.553998526)$ & $\mathbf{p}_{2}=(-0.25117553,0.74347841)$ \\
& $\mathbf{q}_{3}=(0,-0.97986381)$ & $\mathbf{p}_{3}=(-1.03168251,0)$ \\
\hline 2.49999978 & $\mathbf{q}_{1}=(0,0.24972395)$ & $\mathbf{p}_{1}=(1.24210764,0)$ \\
& $\mathbf{q}_{2}=(1.81472444,0.53814821)$ & $\mathbf{p}_{2}=(-0.243722134,0.82104923)$ \\
& $\mathbf{q}_{3}=(0,-1.04886800)$ & $\mathbf{p}_{3}=(-1.04713055,0)$ \\
\hline 3.00000075 & $\mathbf{q}_{1}=(0,0.38988333)$ & $\mathbf{p}_{1}=(1.21778560,0)$ \\
& $\mathbf{q}_{2}=(1.98762581,0.47798374)$ & $\mathbf{p}_{2}=(-0.21039973,0.89083501)$ \\
& $\mathbf{q}_{3}=(0,-1.06770500)$ & $\mathbf{p}_{3}=(-1.07751915,0)$ \\
\hline 3.5 & $\mathbf{q}_{1}=(0,0.55722369)$ & $\mathbf{p}_{1}=(1.19954888,0)$ \\
& $\mathbf{q}_{2}=(2.16184099,0.35108818)$ & $\mathbf{p}_{2}=(-0.14959767,0.95120746)$ \\
& $\mathbf{q}_{3}=(0,-1.03832754)$ & $\mathbf{p}_{3}=(-1.11406449,0)$ \\
\hline
\end{tabular}

Figure 7. Some of the $\tilde{R}_{0}$-symmetric periodic orbits for the modified Hamiltonian $H_{m}$ along the branch connecting the bifurcation points $m=1.459246 \ldots$ and $m=3.945835 \ldots$, together with the corresponding initial data (the missing data are given by $\mathbf{q}_{4}=-S \mathbf{q}_{2}$ and $\left.\mathbf{p}_{4}=S \mathbf{p}_{2}\right)$.

\section{Acknowledgements}

We wish to thank E. Doedel for helpful discussions, and the referees of this paper for their remarks and suggestions which allowed us to considerably improve the presentation. We acknowledge the financial support from the Spanish Ministry of Education through the grant BFM2003-00336. Part of this work was done while the first author had a postdoctoral position at the Departament de Matemàtica Aplicada $i$ Anàlisi of the Universitat de Barcelona. 


\section{References}

Chenciner, A. and Montgomery, R. (2000). "A remarkable periodic solution of the three-body problem in the case of equal masses". Annals of Mathematics, 152:881-901.

Doedel, E., Paffenroth, R., Champneys, A., Fairgieve, F., Kuznetsov, Y.A., Oldeman, B., Sandstede, B., and Wang, X. (2000). AUTO2000: Continuation and bifurcation software for ordinary differential equations". Department of Computer Science, Concordia University, Montreal, Canada, http://sourceforge.net/projects/auto2000/.

Doedel, E., Paffenroth, R., Keller, H., Dichmannm, D., Galán-Vioque, J., and Vanderbauwhede, A. (2003). "Computation of periodic solutions of conservative systems with application to the 3-Body Problem". Int. J. Bifurcation and Chaos, 13:1353-1381.

Ferrario, D. and Terracini, S. (2004). "On the existence of collisionless equivariant minimazers for the classical n-body problem". Invent. Math., 155:305-362

Howison, R. and Meyer, K. (2000). "Doubly-symmetric periodic solutions of the spatial restricted three-body problem". Journal of Diff. Eqns., 163:174-197.

Kapela, T. and Zgliczyński, P. (2003). "The existence of simple choreographies for the $n$-body problem - a computer-assisted proof". Nonlinearity, 16:1899-1918.

Keller, H. (1977). "Numerical solution of bifurcation and nonlinear eigenvalue problems". Applications of Bifurcation Theory, Rabinowitz, P (Editor), pages 359-384, New York. Academic Press.

Lamb, J. S. and Roberts, A. (1998). "Time-reversal symmetry in dynamical systems: a survey". Physica D, 112(1):1-39. Proceedings of the workshop Time-reversal symmetry in dynamical systems.

Loud, W. and Vanderbauwhede, A. (1983). "Functional dependence and boundary value problems with families of solutions". Journal of Diff. Eqns., 47:444-461.

Marchal, C. (2000). "The family $p_{12}$ of the three-body problem-the simplest family of periodic orbits, with twelve symmetries per period". Celestial Mechanics and Dynamical Astronomy, 78:279-298.

Meyer, K. (1981). "Periodic Solutions of the N-Body Problem". Journal of differential equations, 39:2-38.

Meyer, K. (1998). "Continuation of periodic solutions in three dimensions". Physica $D$, 112:310-318.

Meyer, K. R. (1999). "Periodic solutions of the $N$-body problem", volume 1719 of Lecture Notes in Mathematics. Springer-Verlag, Berlin.

Moore, C. (1993). "Braids in classical dynamics" Phys. Rev. Lett.70: 3675-3679.

Muñoz-Almaraz, F. (2003). Continuación y bifurcaciones de órbitas periódicas en sistemas hamiltonianos con simetría. Universidad de Sevilla. (In Spanish).

Muñoz-Almaraz, F., Freire, E., Galán, J., Doedel, E., and Vanderbauwhede, A. (2003). "Continuation of periodic orbits in conservative and Hamiltonian systems". Physica D, 181:1-38.

Muñoz-Almaraz, F., Galán, J., and Freire, E. (2004). "Families of symmetric periodic orbits in the three body problem and the figure eight". Monografías de la Real Academia de Ciencias de Zaragoza, 25:229-240. Avalaible at http://www.unizar.es/acz/05Publicaciones/Monografias/MonografiasPublicadas/ Monografia25/229Munoz.pdf.

Sepulchre, J. and MacKay, R. (1997). "Localized oscilations in conservative networks of weakly coupled autonomous oscillators". Nonlinearity, 10:679-713. 